TRANSACTIONS OF THE

AMERICAN MATHEMATICAL SOCIETY

Volume 353, Number 10, Pages 3895-3918

S 0002-9947(01)02825-2

Article electronically published on May 17, 2001

\title{
SZLENK INDICES AND UNIFORM HOMEOMORPHISMS
}

\author{
G. GODEFROY, N. J. KALTON, AND G. LANCIEN
}

\begin{abstract}
We prove some rather precise renorming theorems for Banach spaces with Szlenk index $\omega_{0}$. We use these theorems to show the invariance of certain quantitative Szlenk-type indices under uniform homeomorphisms.
\end{abstract}

\section{INTRODUCTION}

Classical results about super-reflexive Banach spaces include the Enflo-Pisier renorming theorem $([6],[26])$ and the Heinrich-Mankiewicz theorems on uniform and Lipschitz homeomorphisms [11], from which it follows in particular that the moduli of uniform convexity or uniform smoothness of super-reflexive spaces are invariants for uniform homeomorphisms. This work is an attempt to obtain similar results in the frame of non-super-reflexive spaces. We will in particular relate, in a quantitative way, the Szlenk index with the existence of equivalent UKK*renormings of the space. These results extend and improve results in 18. We will also show that the quantitative dependence on $\epsilon$ of the Szlenk index (when finite) is an invariant under uniform homeomorphisms.

We now turn to a detailed description of our results. In Section 2, the convex Szlenk index $\mathrm{Cz}$ is introduced and compared with the usual Szlenk index Sz from 28. Precise duality formulas, somewhat related to duality between Orlicz spaces, are established, which relate the " $c_{0}$-like" behavior of a Banach space $X$ with the "l 1 -like" behavior of its dual $X^{*}$ (Proposition 2.8). Trees and tree-maps are introduced in Section 3 as a tool to translate estimates on the Szlenk index into geometrical language. Section 4 is devoted to renormings. It was recently shown [18] that if $\mathrm{Sz}(X) \leq \omega_{0}$ then $X$ has an equivalent UKK*-renorming of some power type. We prove more precise results. Trees are an operative tool in the proof of the main renorming theorem (Theorem 4.7) which provides the optimal relation between the behavior of the convex Szlenk index for any given value of $\tau>0$ and the existence of a 2-equivalent norm with the $\mathrm{UKK}^{*}$ property for this value of $\tau$. In Theorem 4.8 we improve the result of [18] mentioned above by giving a precise bound on the power-type. Note however that there is a small loss on the exponent in Theorem 4.8 Such a loss is unavoidable, as is shown by the reflexive example from [18. This also shows that Theorem 4.7 cannot be improved to give a simultaneous renorming which works for all $\tau>0$. Summability of the Szlenk index is shown (Theorem 4.10) to be equivalent to the existence of a constant $K$ with $\tau \cdot \mathrm{Cz}(X, \tau)<K$ for any $\tau \in(0,1)$. Note that Corollary 4.6 asserts that the indices $\mathrm{Cz}$ and $\mathrm{Sz}$ have the same power-type

Received by the editors June 15, 1999 and, in revised form, July 3, 2000.

2000 Mathematics Subject Classification. Primary 46B03, 46B20.

The second author was supported by NSF grant DMS-9870027.

(C)2001 American Mathematical Society 
behavior, at least for $p>1$. We refer to 13 for a presentation of the $\mathrm{UKK}^{*}$ property as asymptotic smoothness, in relation with moduli first introduced in [24]. Section 5 presents applications of these results to uniform homeomorphisms. The main result of this section (Theorem 5.3), whose proof uses the Gorelik principle (Proposition 5.1), asserts in a quantitative way that the existence of equivalent $\mathrm{UKK}^{*}$ norms is invariant under uniform homeomorphisms. The main application of this result (Theorem 5.5) is that the convex Szlenk index is quantitatively invariant under uniform homeomorphisms. This invariance is naturally obtained by a combination of Theorem 5.3 with the renorming Theorem 4.7. Note that, although the class of spaces with separable dual is not stable under uniform homeomorphisms [27], the class of spaces with "very separable" dual (that is, of spaces with Szlenk index $\omega_{0}$ ) is by the above stable under uniform homeomorphisms. It follows also (Theorem 5.6) that a Banach space which is uniformly homeomorphic to $c_{0}$ is an isomorphic predual of $l^{1}$ with summable Szlenk index. We do not know whether a predual of $l^{1}$ with summable Szlenk index is isomorphic to $c_{0}$. Our last application (Theorem 5.8) concerns quotients of $l^{p}$ for $p \in(2, \infty)$.

Some of the results of this work have been announced in [8].

Acknowledgement. This work was initiated when the first and last named authors were visiting the University of Missouri-Columbia in 1997, and was concluded when the second named author was visiting the Université de Besançon in 1999. They are very grateful to these institutions for their hospitality and support. The second author was supported in part by NSF Grant DMS-9870027.

\section{The Szlenk index And PRoperties of NORMS}

We first recall the definition of the Szlenk index and the Szlenk derivation. Suppose $X$ is a separable infinite-dimensional Banach space and $K$ is a weak ${ }^{*}$-compact subset of $X^{*}$. If $\epsilon>0$ we let $\mathcal{V}$ be the set of all weak*-open subsets $V$ of $X^{*}$ such that $\operatorname{diam} V \cap K \leq \epsilon$, and we define the $\epsilon$-interior $\iota_{\epsilon} K=K \backslash \bigcup\{V: V \in \mathcal{V}\}$. (The set $\iota_{\epsilon} K$ is often denoted $K_{\epsilon}^{\prime}$ as in [20], [19]). We then define $\iota_{\epsilon}^{\alpha} K$ for any ordinal $\alpha$ by $\iota_{\epsilon}^{\alpha+1} K=\iota_{\epsilon} \iota_{\epsilon}^{\alpha} K$ and $\iota_{\epsilon}^{\alpha} K=\bigcap_{\beta<\alpha} \iota_{\epsilon}^{\beta} K$ if $\alpha$ is a limit ordinal.

We denote by $B_{X^{*}}$ the closed unit ball of $X^{*}$. We then define $\mathrm{Sz}(X, \epsilon)$ (or $\mathrm{Sz}(\epsilon)$ if no confusion can arise) to be the least countable ordinal $\alpha$ so that $\iota_{\epsilon}^{\alpha} B_{X^{*}}=\emptyset$, if such an ordinal exists. Otherwise we will put $\mathrm{Sz}(X, \epsilon)=\omega_{1}$. The Szlenk index is defined by $\mathrm{Sz}(X)=\sup _{\epsilon>0} \operatorname{Sz}(X, \epsilon)$. We recall that $\mathrm{Sz}(X)<\omega_{1}$ if and only if $X^{*}$ is separable.

Note that $\operatorname{Sz}(X, \epsilon) \geq \epsilon^{-1}$ if $\epsilon>0$, and compactness requires that $\operatorname{Sz}(X, \epsilon)$ is not a limit ordinal. Thus $\operatorname{Sz}(X)=\omega_{0}$ is equivalent to $\operatorname{Sz}(X, \epsilon)<\omega_{0}$ for every $\epsilon>0$, where $\omega_{0}$ denotes the first limit ordinal.

We also introduce an alternative convex Szlenk index. If $K$ is compact and convex we may define $\hat{\iota}_{\epsilon} K=\overline{\mathrm{Co}} \iota_{\epsilon} K$. Then $\mathrm{Cz}(X, \epsilon)$ and $\mathrm{Cz}(X)$ are defined as before, using instead this derivation. Obviously $\mathrm{Cz}(X, \epsilon) \geq \mathrm{Sz}(X, \epsilon)$. On the hand, $\mathrm{Cz}(X)<\omega_{1}$ is equivalent to the separability of $X^{*}$; this follows easily from the weak ${ }^{*}$-dentability of weak ${ }^{*}$-compact sets in separable duals.

Henceforward we will only be interested in cases when $\mathrm{Sz}(X, \epsilon)$ and $\mathrm{Cz}(X, \epsilon)$ are finite. It will therefore be useful to adopt the convention that $\operatorname{Sz}(X, \epsilon)=\infty$ if $\mathrm{Sz}(X, \epsilon) \geq \omega_{0}$, and similarly for $\mathrm{Cz}(X, \epsilon)$.

Following [18], we will say that $X$ admits a summable Szlenk index if there exists a constant $K$ so that $\sum_{i=1}^{n} \epsilon_{i} \leq K$ whenever $\iota_{\epsilon_{1}} \ldots \iota_{\epsilon_{n}} B_{X^{*}} \neq \emptyset$. 
The following lemma is proved in [19], p. 57, or [20]:

Lemma 2.1. If $0<\epsilon, \eta \leq 1$, then $\operatorname{Sz}(X, \epsilon \eta) \leq \operatorname{Sz}(X, \epsilon) \operatorname{Sz}(X, \eta)$.

Note that this implies that $\operatorname{Sz}(X)=\omega_{0}$ if and only if $\operatorname{Sz}(X, \epsilon)<\infty$ for any fixed $0<\epsilon<1$.

Another immediate consequence of this is that if $\mathrm{Sz}(X)=\omega_{0}$, then

$$
\lim _{\epsilon \rightarrow 0} \frac{\log \operatorname{Sz}(X, \epsilon)}{|\log \epsilon|}=p
$$

exists, where $1 \leq p<\infty$. We will call $p=p_{X}$ the Szlenk power type of $X$. We also have that if $\delta>0$ then $\mathrm{Sz}(X, \epsilon) \leq C \epsilon^{-p-\delta}$ for some suitable constant $C$, so that $\mathrm{Sz}(X, \epsilon)$ grows at a power rate. In fact we can also define $p_{X}$ as the infimum of all $q$ such that $\epsilon^{q} \mathrm{Sz}(X, \epsilon)$ is bounded.

Next we note

Lemma 2.2. If $0<\epsilon<1$ and $n \in \mathbb{N}$ are such that $n \epsilon \leq 1$, then:

(1) $\mathrm{Sz}(X, \epsilon)-1 \geq n(\mathrm{Sz}(X, n \epsilon)-1)$.

(2) $\mathrm{Cz}(X, \epsilon)-1 \geq n(\mathrm{Cz}(X, n \epsilon)-1)$.

Proof. Note that if $j<\mathrm{Sz}(X, n \epsilon)$ then, for any $m \geq 2$,

$$
(m-1) B_{X^{*}}+\iota_{n \epsilon}^{j} B_{X^{*}} \subset \iota_{n \epsilon}^{j}\left(m B_{X^{*}}\right) .
$$

Hence $(m-1) B_{X^{*}} \subset \iota_{n \epsilon}^{l} m B_{X^{*}}$, where $l=\mathrm{Sz}(X, n \epsilon)-1$. Iterating, we obtain $\iota_{n \epsilon}^{n l}\left(n B_{X^{*}}\right) \neq \emptyset$, and so $\mathrm{Sz}(X, \epsilon) \geq n l+1$. This implies the result for the Szlenk index, and the convex version is similar.

We will also need the following elementary fact:

Lemma 2.3. Suppose $X$ and $Y$ are isomorphic Banach spaces. Then if $d=$ $d(X, Y)$ we have

$$
\mathrm{Sz}(X, d \epsilon) \leq \mathrm{Sz}(Y, \epsilon) \leq \mathrm{Sz}\left(X, d^{-1} \epsilon\right)
$$

and

$$
\mathrm{Cz}(X, d \epsilon) \leq \mathrm{Cz}(Y, \epsilon) \leq \mathrm{Cz}\left(X, d^{-1} \epsilon\right)
$$

There is one important advantage of the convex Szlenk index which is established in the following lemma. Note that part (2) shows that if the convex Szlenk index is $O\left(\tau^{-1}\right)$, then it is actually summable.

Lemma 2.4. Suppose $X$ is a separable Banach space, $0 \leq \epsilon_{k} \leq 1$ for $1 \leq k \leq N$ and $s=\sum_{i=1}^{N} \epsilon_{i}$.

(1) If $\iota_{\epsilon_{1}} \ldots \iota_{\epsilon_{N}} B_{X^{*}} \neq \emptyset$ and $0<\tau \leq \frac{s}{2 N}$, then

$$
\sum_{\substack{k \geq 0 \\ 2^{k} \tau \leq 1}} 2^{k}\left(\operatorname{Sz}\left(X, 2^{k} \tau\right)-1\right) \geq \frac{s}{2 \tau} .
$$

(2) If $\hat{\iota}_{\epsilon_{1}} \ldots \hat{\iota}_{\epsilon_{N}} B_{X^{*}} \neq \emptyset$ and $0<\tau \leq s /(4 N)$, then

$$
\mathrm{Cz}(X, \tau))-1 \geq \frac{s}{8 \tau} \text {. }
$$


Proof. For the first part, let us write $\alpha_{i} \leq \epsilon_{i} \leq \beta_{i}$, where $\alpha_{i}=2^{k_{i}} \tau$ and $\beta=2^{k_{i}+1} \tau$ with $k_{i}$ a nonnegative integer, or $\alpha_{i}=0$ and $\beta_{i}=\tau$. Then $\beta_{i}=\tau+\sum_{0 \leq j \leq k_{i}} 2^{j} \tau$. Now observe that $\left|\left\{j: \epsilon_{j} \geq 2^{k} \tau\right\}\right|<\mathrm{Sz}\left(X, 2^{k} \tau\right)$, and so

$$
s \leq N \tau+\sum_{\substack{k \geq 0 \\ 2^{k} \tau \leq 1}} 2^{k} \tau\left(\operatorname{Sz}\left(X, 2^{k} \tau\right)-1\right) .
$$

This immediately gives the conclusion of (1).

For the second part, first suppose $C$ is a weak*-compact and convex subset of $X^{*}$. We will argue that if $\epsilon>0$ and $k \in \mathbb{N}$, then $\hat{\iota}_{2 k \epsilon} C \subset \hat{\iota}_{\epsilon}^{k} C$. Indeed, suppose $x^{*} \in \iota_{2 k \epsilon} C$. Then there is a sequence $x_{n}^{*} \in C$ with $\left\|x_{n}^{*}-x^{*}\right\| \geq k \epsilon$ and $x_{n}^{*} \rightarrow x^{*}$ weak ${ }^{*}$. Now for any $n_{1}<n_{2}<n_{3}<\ldots<n_{k}$ we have $\frac{1}{k}\left(x_{n_{1}}^{*}+\cdots+x_{n_{k}}^{*}\right) \in C$. Letting $n_{k} \rightarrow \infty$, we obtain $\frac{1}{k}\left(x^{*}+x_{n_{1}}^{*}+\cdots+x_{n_{k-1}}^{*}\right) \in \iota_{\epsilon} C$. Letting $n_{k-1} \rightarrow \infty$ and repeating, we obtain that $x^{*} \in \iota_{\epsilon}^{k} C$. In particular we observe that $\hat{\iota}_{2 k \epsilon} C \subset \hat{\iota}_{\epsilon}^{k} C$.

Now suppose $\epsilon_{1}, \epsilon_{2}, \ldots, \epsilon_{N}>0$ and $\sum_{k=1}^{N} \epsilon_{k}=s$. Suppose $\tau \leq s / 4 N$, and let $m_{k}=\left[\epsilon_{k} / 2 \tau\right]$. Then

$$
\hat{\iota}_{\epsilon_{1}} \ldots \hat{\iota}_{\epsilon_{N}} B_{X^{*}} \subset \hat{\iota}_{\tau}^{m_{1}+\cdots+m_{N}} B_{X^{*}}
$$

Now

$$
m_{1}+\cdots+m_{N}=\sum_{\epsilon_{k} \geq 2 \tau}\left[\frac{\epsilon_{k}}{2 \tau}\right] \geq \frac{1}{4 \tau} \sum_{\epsilon_{k} \geq 2 \tau} \epsilon_{k} .
$$

But $\tau \leq \frac{s}{4 N}$ yields $\sum_{\epsilon_{k} \leq 2 \tau} \epsilon_{k} \leq \frac{s}{2}$. Hence $m_{1}+\cdots+m_{N} \geq \frac{s}{8 \tau}$.

Let us now turn to renormings. We need the following elementary lemma:

Lemma 2.5. Suppose $X$ is an infinite-dimensional Banach space with separable dual and that $\left(x_{n}^{*}\right)$ is a sequence in $X^{*}$ with $\lim _{n \rightarrow \infty} x_{n}^{*}=0$ weak* . Then there is a sequence $\left(x_{n}\right)$ in $X$ with $\left\|x_{n}\right\| \leq 1, \lim _{n \rightarrow \infty} x_{n}=0$ weakly and

$$
\liminf \left\langle x_{n}, x_{n}^{*}\right\rangle \geq \frac{1}{2} \liminf \left\|x_{n}^{*}\right\| .
$$

Proof. We observe that by Lemma 2.3 of [17] we have

$$
\liminf _{n \rightarrow \infty} d\left(x_{n}^{*}, F\right) \geq \frac{1}{2} \liminf _{n \rightarrow \infty}\left\|x_{n}^{*}\right\|
$$

if $F$ is any finite-dimensional subspace of $X^{*}$. Hence, since $X^{*}$ is separable we find an increasing sequence (not necessarily strictly increasing) of finite-dimensional subspaces $\left(F_{n}\right)$ so that $\bigcup F_{n}$ is dense in $X^{*}$ and $\lim \inf d\left(x_{n}^{*}, F_{n}\right) \geq \frac{1}{2} \liminf _{n \rightarrow \infty}\left\|x_{n}^{*}\right\|$.

There exist $x_{n} \in X$ with $\left\|x_{n}\right\|=1, x_{n} \in F_{n}^{\perp}$ and $\left\langle x_{n}, x_{n}^{*}\right\rangle>d\left(x_{n}^{*}, F_{n}\right)-\frac{1}{n}$. This gives the conclusion.

Proposition 2.6. Suppose $X$ is a separable Banach space and $0<\sigma, \tau<1$. Consider the following statements:

1. If $x^{*} \in X^{*}$ with $\left\|x^{*}\right\|=1, \lim x_{n}^{*}=0$ weak $k^{*}$ with $\lim _{n \rightarrow \infty}\left\|x_{n}^{*}\right\|=\tau$, then

$$
\liminf _{n \rightarrow \infty}\left\|x^{*}+x_{n}^{*}\right\| \geq 1+\sigma \tau .
$$

2. $X^{*}$ is separable, and if $x \in X$ with $\|x\|=1, \lim x_{n}=0$ weakly with $\lim \left\|x_{n}\right\|=$ $\sigma$, then

$$
\limsup _{n \rightarrow \infty}\left\|x+x_{n}\right\| \leq 1+\sigma \tau
$$


3. $X^{*}$ is separable, and if $x^{*} \in X^{*}$ with $\left\|x^{*}\right\|=1, \lim x_{n}^{*}=0$ weak $k^{*}$ with $\lim _{n \rightarrow \infty}\left\|x_{n}^{*}\right\|=6 \tau$, then

$$
\liminf _{n \rightarrow \infty}\left\|x^{*}+x_{n}^{*}\right\| \geq 1+\sigma \tau .
$$

Then $(1) \Rightarrow(2) \Rightarrow(3)$. Furthermore (1) implies that

$$
\mathrm{Sz}(X, 2 \tau) \leq \mathrm{Cz}(X, 2 \tau) \leq \sigma^{-1} \tau^{-1}+1 .
$$

(In particular, if $2 \tau<1$ then $\mathrm{Sz}(X)=\omega_{0}$.)

Proof. Note that (1) implies that $\iota_{2 \tau} B_{X^{*}} \subset(1-\sigma \tau) B_{X^{*}}$, and this immediately yields $\mathrm{Cz}(X, 2 \tau) \leq \sigma^{-1} \tau^{-1}+1$. Then the last statement follows from Lemma 2.1

First assume (1) holds and $x, x_{n}$ are chosen as in (2). It is enough to show it is impossible that $\lim _{n \rightarrow \infty}\left\|x+x_{n}\right\|>1+\sigma \tau$. Suppose this holds. Then we can pick $y_{n}^{*} \in B_{X^{*}}$ with $\lim _{n \rightarrow \infty}\left\langle x+x_{n}, y_{n}^{*}\right\rangle>1+\sigma \tau$. Passing to a subsequence, we can suppose $y_{n}^{*}$ converges weak ${ }^{*}$ to some $x^{*} \in B_{X^{*}}$, and then put $x_{n}^{*}=y_{n}^{*}-x^{*}$. We can assume that $\lim _{n \rightarrow \infty}\left\|x_{n}^{*}\right\|=\theta$ exists.

If $\theta \leq \tau$ then

$$
\lim _{n \rightarrow \infty}\left\langle x+x_{n}, x^{*}+x_{n}^{*}\right\rangle \leq\left\langle x, x^{*}\right\rangle+\sigma \tau \leq 1+\sigma \tau .
$$

On the other hand, if $\theta>\tau$ then we have, using the convexity of the norm and (1),

$$
1 \geq \liminf _{n \rightarrow \infty}\left\|x^{*}+x_{n}^{*}\right\| \geq\left\|x^{*}\right\|+\sigma \theta .
$$

Hence $\left\|x^{*}\right\| \leq 1-\sigma \theta$, and so

$$
\lim _{n \rightarrow \infty}\left\langle x+x_{n}, x^{*}+x_{n}^{*}\right\rangle \leq 1-\sigma \theta+\sigma \theta=1 .
$$

This gives us the required contradiction.

Assume (2) holds. Suppose that $x^{*}, x_{n}^{*}$ are chosen as in (3). It will be enough to show the conclusion for some subsequence.

Then, given $\epsilon>0$, we can choose $x \in X$ with $\|x\|=1$ and $x^{*}(x)>1-\epsilon$. Now by Lemma 2.5, we can, by passing to a subsequence, assume there exists a weakly null sequence $x_{n} \in X$ with $\left\|x_{n}\right\|=1$ and $\liminf x_{n}^{*}\left(x_{n}\right) \geq 3 \tau$. Now

$$
\liminf \left\langle x+\sigma x_{n}, x^{*}+x_{n}^{*}\right\rangle \geq 1-\epsilon+3 \sigma \tau,
$$

and so by (2)

$$
1-\epsilon+3 \sigma \tau \leq(1+\sigma \tau) \liminf \left\|x^{*}+x_{n}^{*}\right\| .
$$

Hence letting $\epsilon \rightarrow 0$ we have

$$
\liminf \left\|x^{*}+x_{n}^{*}\right\| \geq \frac{1+3 \sigma \tau}{1+\sigma \tau} \geq 1+\sigma \tau .
$$

Proposition 2.7. Let $X$ be a separable Banach space not containing a copy of $\ell_{1}$, and suppose $0<\sigma<1$ and $0<\tau<\frac{1}{2}$. Suppose that whenever $x \in X$ with $\|x\|=1$ and $\lim x_{n}=0$ weakly with $\lim _{n \rightarrow \infty}\left\|x_{n}\right\|=\sigma$, then

$$
\limsup _{n \rightarrow \infty}\left\|x+x_{n}\right\| \leq 1+\sigma \tau .
$$

Then $X^{*}$ contains no proper norming subspaces and hence is separable. 
Proof. If $X^{*}$ contains a proper norming subspace, there is $x^{* *} \in X^{* *}$ of norm one such that $\left\{x^{*} \in X^{*}: x^{* *}\left(x^{*}\right)=0\right\}$ is a norming subspace of $X^{*}$. By the OdellRosenthal theorem [25] there is a weakly Cauchy sequence $\left(x_{n}\right)$ in $X$ with $\left\|x_{n}\right\|=1$ and $x_{n} \rightarrow x^{* *}$ weak* .

If $m \in \mathbb{N}$, then for any $\epsilon>0$ and for each $n>m$ we can choose $e^{*} \in X^{*}$ with $\left\|e^{*}\right\|=1, x^{* *}\left(e^{*}\right)=0$ and $e^{*}\left(x_{m}+\frac{1}{2} \sigma x_{n}\right)>\left\|x_{m}+\frac{1}{2} \sigma x_{n}\right\|-\epsilon$. Then we can find $k(n)>n$ so that $e^{*}\left(\sigma x_{k(n)}\right)<\epsilon$. We conclude that

$$
\left\|x_{m}+\frac{1}{2} \sigma\left(x_{n}-x_{k(n)}\right)\right\|>\left\|x_{m}+\frac{1}{2} \sigma x_{n}\right\|-2 \epsilon .
$$

Letting $n \rightarrow \infty$, since $\left(x_{n}-x_{k(n)}\right) \rightarrow 0$ weakly we have

$$
\limsup _{n \rightarrow \infty}\left\|x_{m}+\frac{1}{2} \sigma x_{n}\right\| \leq 1+\sigma \tau+2 \epsilon .
$$

Thus

$$
\left\|x_{m}+\frac{1}{2} \sigma x^{* *}\right\| \leq 1+\sigma \tau
$$

Then as $m \rightarrow \infty$ we have $1+\frac{1}{2} \sigma \leq 1+\sigma \tau$, which is a contradiction.

At this point we introduce some terminology. Let $f, g$ be continuous monotone increasing functions on $[0,1]$ which satisfy $f(0)=g(0)=0$. We will say that $f$ $C$-dominates $g$ if $f(\tau) \geq g(\tau / C)$ for every $0 \leq \tau \leq 1$. We will say that $f, g$ are $C$-equivalent if $f C$-dominates $g$ and $g C$-dominates $f$.

For any such monotone increasing function $f$ we denote by $f^{*}$ its dual Young's function, i.e.

$$
f^{*}(s)=\sup \{s t-f(t): 0 \leq t \leq 1\} .
$$

Notice that if $f C$-dominates $g$ then $g^{*} C$-dominates $f^{*}$. Note also that $f^{*}$ is a convex function.

Now, if $X$ is any separable Banach space, for $0 \leq \sigma \leq 1$ we define $\rho(\sigma)=\rho_{X}(\sigma)$ to be the least constant so that

$$
\limsup _{n \rightarrow \infty}\left\|x+x_{n}\right\| \leq 1+\rho_{X}(\sigma)
$$

whenever $\|x\|=1, \lim x_{n}=0$ weakly and $\limsup _{n \rightarrow \infty}\left\|x_{n}\right\| \leq \sigma$. We define $\theta(\tau)=$ $\theta_{X}(\tau)$ for $0 \leq \tau \leq 1$ to be the greatest constant so that

$$
\liminf _{n \rightarrow \infty}\left\|x^{*}+x_{n}^{*}\right\| \geq 1+\theta_{X}(\tau)
$$

whenever $x^{*}, x_{n}^{*} \in X^{*},\left\|x^{*}\right\|=1, \lim x_{n}^{*}=0$ weak $^{*}$ and $\liminf _{n \rightarrow \infty}\left\|x_{n}^{*}\right\| \geq \tau$.

We then define $\varphi(\sigma)$ by

$$
\varphi(\sigma)=\inf \left\{\rho_{Y}(\sigma): d(X, Y) \leq 2\right\}
$$

and $\psi(\tau)=\sup \left\{\theta_{Y}(\tau): d(X, Y) \leq 2\right\}$

We can now summarize Proposition 2.6 and Proposition 2.7:

Proposition 2.8. Let $X$ be a separable Banach space not containing $\ell_{1}$. Then:

1. Each of the functions $\rho(t) / t, \theta(t) / t, \varphi(t) / t$ and $\psi(t) / t$ is monotone increasing on $(0,1)$.

2. $\theta$ is 4-equivalent to $\rho^{*}$ and $\psi$ is 4-equivalent to $\varphi^{*}$.

3. $\rho$ is 8-equivalent to $\theta^{*}$ and $\varphi$ is 8-equivalent to $\psi^{*}$. 
4. If $0<t \leq 1$ then

$$
\mathrm{Cz}(X, t) \leq 1+\frac{1}{\psi(t / 4)} .
$$

Proof. (1) follows trivially from convexity considerations.

(2) It follows from Proposition 2.7 that if $X^{*}$ is not separable, then $\rho^{*}(\tau)=0$ for all $0 \leq \tau \leq \frac{1}{2}$; and then it is obvious that $\theta 2$-dominates $\rho^{*}$. If $X^{*}$ is separable, we pick $\left(x_{n}^{*}\right)$ in $X^{*}$ such that $\liminf _{n \rightarrow \infty}\left\|x_{n}^{*}\right\| \geq \tau$ and

$$
\liminf _{n \rightarrow \infty}\left\|x^{*}+x_{n}^{*}\right\|=1+\theta_{X}(\tau)
$$

By Lemma 2.5 there is a weakly null sequence $\left(x_{n}\right)$ with $\lim \sup _{n \rightarrow \infty}\left\|x_{n}\right\| \leq \sigma$ and $\lim x_{n}^{*}\left(x_{n}\right)=\sigma \tau / 2$. It follows easily that

$$
1+\sigma \tau / 2 \leq(1+\rho(\sigma))(1+\theta(\tau)),
$$

so that

$$
\sigma \tau / 2 \leq \rho(\sigma)+\theta(\tau)+\rho(\sigma) \theta(\tau) .
$$

Now since $\theta(\tau) \leq 1$ we obtain

$$
\theta(\tau) \geq \sigma \tau / 2-2 \rho(\sigma) .
$$

Since $\sigma$ is arbitrary, it follows that

$$
\theta(\tau) \geq 2 \rho^{*}(\tau / 4) .
$$

Hence $\theta$ 4-dominates $\rho^{*}$.

The same considerations show that $\rho$ 4-dominates $\theta^{*}, \varphi$ 4-dominates $\psi^{*}$ and $\psi$ 4-dominates $\varphi^{*}$.

Now if $0<\tau<1$ pick $\sigma=2 \theta(\tau / 2) / \tau$. Then by Proposition 2.6] we have $\rho(\sigma) \leq$ $\frac{1}{2} \sigma \tau$. Hence

$$
\theta(\tau / 2)=\frac{1}{2} \sigma \tau \leq \sigma \tau-\rho(\sigma) \leq \rho^{*}(\tau) .
$$

Thus $\rho^{*}$ 2-dominates $\theta$. The proof for $\psi$ in place of $\theta$ and $\varphi$ in place of $\rho$ is similar.

(3) We deduce from (2) that $\theta^{*}$ is 4-equivalent to $\rho^{* *}$. Next let

$$
\tilde{\rho}(t)=\int_{0}^{t} \frac{\rho(\tau)}{\tau} d \tau
$$

Then $\rho(t / 2) \leq \tilde{\rho}(t) \leq \rho(t)$. Hence since $\tilde{\rho}$ is convex we have $\rho^{* *}(t) \geq \tilde{\rho}(t) \geq \rho(t / 2)$. Hence $\rho$ is 2-equivalent to $\rho^{* *}$. The argument for $\varphi$ and $\psi$ is similar.

(4) This is an immediate deduction from Proposition 2.6 and Lemma 2.3.

\section{TREes AND TREe-MAPS}

Consider the set $\mathcal{F} \mathbb{N}$ of all finite subsets of $\mathbb{N}$ with the following partial order. If $a=\left\{n_{1}, n_{2}, \ldots, n_{k}\right\}$ where $n_{1}<n_{2}<\ldots<n_{k}$ and $b=\left\{m_{1}, m_{2}, \ldots, m_{l}\right\}$ where $m_{1}<m_{2}<\cdots<m_{l}$, then $a \leq b$ if and only if $k \leq l$ and $m_{i}=n_{i}, 1 \leq i \leq k$ (i.e. $a$ is an initial segment of $b$ ). We say that $b$ is a successor of $a$ if $|b|=|a|+1$ and $a \leq b$; the collection of successors of $a$ is denoted by $a+$. If $a \neq \emptyset$ then $a-$ denotes the unique predecessor of $a$, i.e. $a$ is a successor of $a-$. Let $S$ be a subset of $\mathcal{F N}$. We will say that $S$ is a full tree if the following three conditions hold:

1. $\emptyset \in S$.

2. Each $a \in S$ has infinitely many successors in $S$.

3. If $a \in S$ and $\emptyset \neq a \in S$, then $a-\in S$. 
It is easy to see that any full tree is isomorphic as an ordered set to $\mathcal{F}$. If $S$ is any full tree, we will say that a sequence $\beta=\left\{a_{n}\right\}_{n=0}^{\infty}$ is a branch of $S$ if $a_{n} \in S$ for all $n, a_{0}=\emptyset$ and $a_{n+1}$ is a successor of $a_{n}$ for all $n \geq 0$.

Now let $V$ be a vector space. We define a tree-map in $V$ to be a map $a \mapsto x_{a} \in V$ defined on a full tree $S$ with the properties that $x_{\emptyset}=0$ and for every branch $\beta$ the set $\left\{a: x_{a} \neq 0: a \in \beta\right\}$ is finite. Given any tree-map, we define a height function $h$ which assigns to each $a$ a countable ordinal; to do this we define $h(a)=0$ if $x_{b}=0$ for $b \geq a$, and then inductively $h(a)$ is defined by $h(a) \leq \eta$ if and only if $h(b)<\eta$ for every $b>a$. The height of the tree-map is defined to be $h(\emptyset)$. Note that the tree-map $a \mapsto x_{a}$ has finite height $m \leq n$ if and only if $x_{a}=0$ whenever $|a|>n$. For a recent work on trees in Banach spaces we refer to [2].

The following easy lemma is a restatement of the fact that certain types of games (which are not used in this paper) are determined.

Lemma 3.1. Suppose $\left(x_{a}\right)_{a \in S}$ is a tree-map in $V$ and that $A$ is any subset of $V$. Then either there is a full tree $T \subset S$ so that $\sum_{a \in \beta} x_{a} \in A$ for every branch $\beta \subset T$, or there is a full tree $T \subset S$ so that $\sum_{a \in \beta} x_{a} \notin A$ for every branch $\beta \subset T$.

Proof. For each countable ordinal $\eta$ we define a subset $B_{\eta}$ of $\{a \in S: h(a)=\eta\}$ as follows. If $\eta=0$ let $a \in B_{0}$ if $\sum_{b \leq a} x_{a} \in A$. Then inductively if $h(a)=\eta$ we say $a \in B_{\eta}$ if $a$ has infinitely many successors $b$ with $b \in B_{h(b)}$. Let $B=\bigcup_{\eta} B_{\eta}$. If $\emptyset \in B$ then an easy induction argument produces a full tree $T \subset S$ with $\sum_{a \in \beta} x_{a} \in A$ for every $\beta \subset T$. Otherwise the set $T=S \backslash B$ is a full tree with the property that $\sum_{a \in \beta} x_{a} \notin A$ for every $\beta \subset T$.

We now consider tree-maps with values in a Banach space $X$.

Lemma 3.2. Suppose $\left(x_{a}\right)_{a \in S}$ is a bounded tree-map in $X$ of finite height $n$. Then, given $\delta>0$ we can find a full tree $T \subset S$ and $\epsilon_{1}, \ldots, \epsilon_{n} \geq 0$ so that if $a \in T$ and $|a|=k \leq n$ then $\epsilon_{k} \leq\left\|x_{a}\right\| \leq \epsilon_{k}+\delta$.

Proof. One easy way to prove this is to consider $V=\mathbb{R}^{n}$ with canonical basis $e_{1}, \ldots, e_{n}$, and then the tree-map $u_{a}=\left\|x_{a}\right\| e_{k}$ if $|a|=k \leq n$ and $u_{a}=0$ if $|a|>n$. The lemma follows from the Heine-Borel theorem and repeated applications of Lemma 3.1 .

If $\tau$ is a topology on $X$ (e.g. the weak topology or for dual spaces the weak*topology) we say that a tree-map $\left(x_{a}\right)_{a \in S}$ is $\tau-$ null if for every $a \in S$ the set $\left\{x_{b}\right\}_{b \in a+}$ is a $\tau$-null sequence.

Lemma 3.3. Suppose $X$ is a Banach space and $\left(x_{a}\right)_{a \in S}$ is a weakly null tree-map in $X$ and $\left(x_{a}^{*}\right)_{a \in S}$ is a weak $k^{*}$ null tree map in $X^{*}$. Then for any $\delta>0$ there is a full tree $T \subset S$ so that for any branch $\beta \subset T$ we have

$$
\left|\left\langle\sum_{a \in \beta} x_{a}, \sum_{a \in \beta} x_{a}^{*}\right\rangle-\sum_{a \in \beta}\left\langle x_{a}, x_{a}^{*}\right\rangle\right| \leq \delta .
$$

Proof. Let $T$ be the set of $a$ so that if $b<c \leq a$ then $\left|\left\langle x_{b}, x_{c}^{*}\right\rangle\right|,\left|\left\langle x_{c}, x_{b}^{*}\right\rangle\right| \leq$ $|c|^{-1} 2^{-|c|-1} \delta$. It is not difficult to see that this is a full tree, and that if $\beta$ is a branch in this tree then

$$
\left|\left\langle\sum_{a \in \beta} x_{a}, \sum_{a \in \beta} x_{a}^{*}\right\rangle-\sum_{a \in \beta}\left\langle x_{a}, x_{a}^{*}\right\rangle\right|=\left|\sum_{b<a} \sum_{a \in \beta}\left\langle x_{a}, x_{b}^{*}\right\rangle+\left\langle x_{b}, x_{a}^{*}\right\rangle\right| \leq \delta .
$$


Proposition 3.4. Let $X$ be a separable Banach space. In order that

$$
\iota_{\epsilon_{1}} \iota_{\epsilon_{2}} \ldots \iota_{\epsilon_{n}} B_{X^{*}} \neq \emptyset
$$

it is necessary that there exists a weak ${ }^{*}$-null tree-map $\left(x_{a}^{*}\right)_{a \in S}$ in $X^{*}$ with $\left\|x_{a}^{*}\right\| \geq$ $\frac{1}{4} \epsilon_{|a|}$ for $1 \leq|a| \leq n$ and so that $\left\|\sum_{a \in \beta} x_{a}^{*}\right\| \leq 1$ for every branch $\beta$, and sufficient that there exists a weak ${ }^{*}$-null tree-map $\left(x_{a}^{*}\right)_{a \in S}$ in $X^{*}$ with $\left\|x_{a}^{*}\right\| \geq \epsilon_{|a|}$ for $1 \leq|a| \leq$ $n$ and so that $\left\|\sum_{a \in \beta} x_{a}^{*}\right\| \leq 1$ for every branch $\beta$.

Proof. First assume $\iota_{\epsilon_{1}} \iota_{\epsilon_{2}} \ldots \iota_{\epsilon_{n}} B_{X^{*}}=K \neq \emptyset$. Then

$$
0 \in \frac{1}{2} K+\frac{1}{2} B_{X^{*}} \subset \iota_{\epsilon_{1} / 2} \ldots \iota_{\epsilon_{n} / 2} B_{X^{*}} .
$$

Now there exists a sequence $x_{k}^{*}$ converging to 0 weak $^{*}$ so that $\left\|x_{k}^{*}\right\| \geq \frac{1}{4} \epsilon_{1}$ and $x_{k}^{*} \in \iota_{\epsilon_{2} / 2} \iota_{\epsilon_{3} / 2} \ldots \iota_{\epsilon_{n} / 2} B_{X^{*}}$, since otherwise there is a weak* ${ }^{*}$-open neighborhood of 0 relative to $\iota_{\epsilon_{2} / 2} \iota_{\epsilon_{3} / 2} \ldots \iota_{\epsilon_{n} / 2} B_{X^{*}}$ of diameter less than $\epsilon_{1} / 2$. Then for each $k \in \mathbb{N}$ we find a sequence $\left(x_{k_{1}, k_{2}}^{*}\right)_{k_{2}>k_{1}}$ so that $\lim _{k_{2}} x_{k_{1}, k_{2}}^{*}=0$ weak $^{*},\left\|x_{k_{1}, k_{2}}^{*}\right\| \geq \frac{1}{4} \epsilon_{2}$ and $x_{k_{1}}^{*}+x_{k_{1}, k_{2}}^{*} \in \iota_{\epsilon_{3} / 2} \ldots \iota_{\epsilon_{n} / 2} B_{X^{*}}$. This procedure can then be iterated to define $x_{a}^{*}$ if $|a| \leq n$. Setting $x_{a}^{*}=0$ if $|a|>n$, we obtain the desired tree-map.

The converse is equally easy. Obviously, if $\left(x_{a}^{*}\right)_{a \in S}$ is the given tree-map, then we have $\left\|\sum_{a \leq b} x_{a}^{*}\right\| \leq 1$ for all $b$. It then follows inductively that $\sum_{b \leq a} x_{b}^{*} \in$ $\iota_{\epsilon_{k+1}} \iota_{\epsilon_{k+2}} \cdots \iota_{\epsilon_{n}} B_{X^{*}}$ whenever $|a|=k$. Setting $k=0$ gives the result.

\section{4. $\mathrm{UKK}^{*}$-RENORMINGS}

Suppose $X$ is a separable Banach space. If $\sigma>0$ we define $N=N(\sigma)$ to be the least integer $N$ so that there exists a weakly null tree-map $\left(x_{a}\right)_{a \in S}$ in $X$ of height $N+1$ such that $\left\|x_{a}\right\| \leq \sigma$ for every $a \in S$ and $\left\|\sum_{a \in \beta} x_{a}\right\|>1$ for every branch $\beta$. (We put $N(\sigma)=\infty$ if no such integer exists.)

Notice that $N(\sigma)>\sigma^{-1}-1$.

Remark. It follows from Lemma 3.1 that if $k \leq N(\sigma)$ then for every weakly null tree-map $\left(x_{a}\right)_{a \in S}$ of height $k$ and such that $\left\|x_{a}\right\| \leq \sigma$ for all $a \in S$ there is a full tree $T \subset S$ so that $\left\|\sum_{a \in \beta} x_{a}\right\| \leq 1$ for every branch.

We refer to [4] and references therein for the uniform Kadec-Klee property and its dual version. Here we denote by $\mathrm{UKK}^{*}$ what was named there weak-star UKK. According to the more sugestive terminology introduced in [13], spaces with the UKK $^{*}$ property should be called asymptotically smooth spaces. Also, we consider it as a property of a Banach space which can be checked on its dual, rather than a property of a dual space which refers to a given predual. Our main reason for introducing the quantity $N(\sigma)$ is to obtain a renorming of the space $X$ with an approximate $\mathrm{UKK}^{*}$-condition.

Theorem 4.1. Suppose $X$ is a separable Banach space. Then, for any $\sigma>0$, if $N(\sigma)<\infty$ there is a norm $|\cdot|$ on $X$ satisfying $\frac{1}{2}\|x\| \leq|x| \leq\|x\|$ and

$$
\limsup _{n \rightarrow \infty}\left|x+x_{n}\right| \leq 1+\frac{1}{N(\sigma)}
$$

whenever $|x|=1$ and $\left(x_{n}\right)$ is a sequence satisfying

$$
\lim _{n \rightarrow \infty}\left|x_{n}\right|=\frac{1}{2} \sigma \quad \text { and } \quad \lim _{n \rightarrow \infty} x_{n}=0
$$

weakly. 
Proof. Define $f_{0}(x)=\|x\|$, and then for $k>0$ define $f_{k}(x)$ to be the infimum of all $\lambda>0$ so that whenever $\left(x_{a}\right)_{a \in S}$ is a weakly null tree-map of height $k$ with $\left\|x_{a}\right\| \leq \sigma$ for all $a \in S$ then there is a full subtree $T \subset S$ so that $\left\|x+\sum_{a \in \beta} x_{a}\right\| \leq \lambda$ for every branch $\beta$. We observe first that $\left(f_{k}(x)\right)_{k=0}^{\infty}$ is an increasing sequence, and that $f_{k}(x)=f_{k}(-x)$. Next notice that

$$
\left|f_{k}(x)-f_{k}(y)\right| \leq\|x-y\|
$$

by an elementary calculation, which we omit. We also observe that $f_{N}(x) \leq\|x\|+1$, where $N=N(\sigma)$.

We next claim that $f_{k}$ is convex. Indeed, let $u=t x+(1-t) y$, where $0<t<1$. Suppose $\lambda>f_{k}(x)$ and $\mu>f_{k}(y)$. Let $\left(x_{a}\right)_{a \in S}$ be any weakly null tree-map of height $k$ with $\left\|x_{a}\right\| \leq \sigma$ for all $a \in S$.

Then we can find a full subtree $T_{1} \subset S$ so that for every branch $\beta$ we have

$$
\left\|x+\sum_{a \in \beta} x_{a}\right\| \leq \lambda
$$

and then a full subtree $T_{2} \subset T_{1}$ so that for every branch $\beta \subset T_{2}$

$$
\left\|y+\sum_{a \in \beta} x_{a}\right\| \leq \mu
$$

Obviously for every branch $\beta \subset T_{2}$

$$
\left\|u+\sum_{a \in \beta} x_{a}\right\| \leq t \lambda+(1-t) \mu,
$$

so that $f_{k}(u) \leq t \lambda+(1-t) \mu$.

Next we note that if $\left\|x_{n}\right\| \leq \sigma$ and $\lim _{n \rightarrow \infty} x_{n}=0$ weakly, then

$$
\limsup f_{k}\left(x+x_{n}\right) \leq f_{k+1}(x)
$$

for all $k \geq 0$. Indeed, for $k=0$ this is obvious. If $k>0$, assume that $\lambda<$ $\lim \sup _{n \rightarrow \infty} f_{k}\left(x+x_{n}\right)$. By passing to a subsequence we can suppose $\lambda<f_{k}\left(x+x_{n}\right)$ for every $n$. Then for each $n$ there is a weakly null tree-map $\left(y_{a}^{(n)}\right)_{a \in S_{n}}$ of height $k$ so that $\left\|y_{a}^{(n)}\right\| \leq \sigma$ for all $a \in S_{n}$ and

$$
\left\|x+x_{n}+\sum_{a \in \beta} y_{a}^{(n)}\right\|>\lambda
$$

for every branch $\beta \subset S_{n}$.

Now let $T$ be the tree consisting of all sets $\left\{m_{1}, \ldots, m_{l}\right\}$, where $m_{1}<m_{2}<$ $\cdots<m_{l}$, such that if $l>1$ then $\left\{m_{2}, \ldots, m_{l}\right\} \in S_{m_{1}}$. We define a weakly null tree-map of height $k+1$ by

$$
z_{m_{1}, \ldots, m_{l}}= \begin{cases}x_{m_{1}} & \text { if } l=1 \\ y_{m_{2}, \ldots, m_{l}}^{\left(m_{1}\right)} & \text { if } l>1 .\end{cases}
$$

Then for every branch $\beta \subset T$ we have

$$
\left\|x+\sum_{a \in \beta} z_{a}\right\|>\lambda,
$$

so that $f_{k+1}(x) \geq \lambda$. This implies our claim. 
Now let us set $g(x)=\frac{1}{N} \sum_{k=0}^{N-1} f_{k}(x)$. Then $g$ is convex, and $\|x\| \leq g(x) \leq$ $\|x\|+1$. Further, if $\left(x_{n}\right)$ is weakly null with $\left\|x_{n}\right\| \leq \sigma$ for all $n$, then

$$
\begin{aligned}
\limsup _{n \rightarrow \infty} g\left(x+x_{n}\right) & \leq \frac{1}{N} \sum_{k=1}^{N} f_{k}(x) \\
& \leq g(x)+\frac{1}{N}\left(f_{N}(x)-f_{0}(x)\right) \\
& \leq g(x)+\frac{1}{N} .
\end{aligned}
$$

Let $|\cdot|$ be the Minkowski functional of the set $\{x: g(x) \leq 2\}$. Then it is clear that $\frac{1}{2}|| x\|\leq|x| \leq\| x \|$. Suppose $|x|=1$ and $\left(x_{n}\right)$ is a weakly null sequence with $\left|x_{n}\right| \leq \frac{1}{2} \sigma$. Then $\left\|x_{n}\right\| \leq \sigma$. Thus

$$
\limsup _{n \rightarrow \infty} g\left(x+x_{n}\right) \leq g(x)+\frac{1}{N} \leq 2+\frac{1}{N} .
$$

Now $g(0)<1$, and so from the convexity of $g$ it follows that

$$
\limsup _{n \rightarrow \infty} g\left(\frac{N}{N+1}\left(x+x_{n}\right)\right)<2
$$

whence

$$
\limsup _{n \rightarrow \infty}\left|x+x_{n}\right| \leq 1+\frac{1}{N} .
$$

Theorem 4.2. Suppose $X$ is a separable Banach space. Suppose $\sigma>0$ is such that $N(\sigma)=\infty$. Then, for any $\epsilon>0$ there is a norm $|\cdot|$ on $X$ satisfying $\frac{1}{2}\|x\| \leq|x| \leq\|x\|$ and

$$
\limsup _{n \rightarrow \infty}\left|x+x_{n}\right| \leq 1+\epsilon
$$

whenever $|x|=1$ and $\left(x_{n}\right)$ is a sequence satisfying

$$
\lim _{n \rightarrow \infty}\left|x_{n}\right|=\frac{1}{2} \sigma \quad \text { and } \quad \lim _{n \rightarrow \infty} x_{n}=0
$$

weakly.

Proof. The proof is almost identical to the proof of the preceding Theorem 4.1. except that one considers $g_{m}(x)=\frac{1}{m} \sum_{k=0}^{m-1} f_{k}(x)$ for arbitrarily large choices of $m$. We omit the details.

Notice that the preceding two theorems allow us to say, in the language of Section 2 that $N^{-1}$ 2-dominates $\varphi$, where $N^{-1}(\sigma)=(N(\sigma))^{-1}$.

We now turn to the problem of relating the function $N(\sigma)$ to the convex Szlenk index.

Lemma 4.3. Suppose $0<\sigma<1$. If $N=N(\sigma)$, there exist $0<\epsilon_{1}, \ldots, \epsilon_{N+1}<1$ so that $\sum_{k=1}^{N+1} \epsilon_{k}>\frac{1}{3} \sigma^{-1}$ and $\iota_{\epsilon_{1}} \iota_{\epsilon_{2}} \ldots \iota_{\epsilon_{N+1}} B_{X^{*}} \neq \emptyset$.

Proof. Suppose $\left(x_{a}\right)_{a \in S}$ is a weakly null tree-map of height $N+1$ with $\left\|x_{a}\right\| \leq \sigma$ and so that $\left\|\sum_{a \in \beta} x_{a}\right\|>1$ for every branch $\beta$.

Fix $\delta>0$ so that $(2 N+3) \delta<\frac{1}{3}$. For any $a \in S$ with $|a|=N+1$ we choose $y_{a}^{*}$ with $\left\|y_{a}^{*}\right\|=1$ and $\left\langle\sum_{b \leq a} x_{b}, y_{a}^{*}\right\rangle=\left\|\sum_{b \leq a} x_{b}\right\|$. If $|a|>N$, set $y_{a}^{*}=y_{b}^{*}$, where 
$|b|=N$ and $b \leq a$. We now define $y_{a}^{*}$ by backwards induction, so that for each $a$,

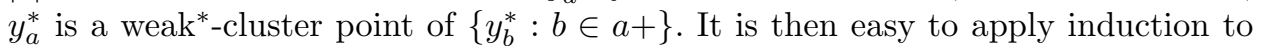
produce a full tree $T \subset S$ so that $\lim _{b \in a+} y_{b}^{*}=y_{a}^{*}$.

Now let $x_{a}^{*}=y_{a}^{*}-y_{a-}^{*}$ when $|a| \geq 1$, and $x_{\emptyset}^{*}=0$, so that $\left(x_{a}^{*}\right)$ is a weak ${ }^{*}$-null tree-map in $X^{*}$ of height $N$. Let $y^{*}=y_{\emptyset}^{*}$, so that $\left\|y^{*}\right\| \leq 1$. We thus have, on every branch $\beta$ of $T$,

$$
\left\|y^{*}+\sum_{a \in \beta} x_{a}^{*}\right\|=1
$$

and so

$$
\left\|\sum_{a \in \beta} x_{a}^{*}\right\| \leq 2 .
$$

Now using Lemma 3.2 we can pass to a further full tree $T_{1} \subset T$ so that for suitable $\epsilon_{1}, \ldots, \epsilon_{N+1}$ we have $2 \epsilon_{k} \leq\left\|x_{a}^{*}\right\| \leq 2 \epsilon_{k}+\delta$ if $|a|=k$ for $1 \leq k \leq N$. Then by Proposition 3.4 we have that $\iota_{\epsilon_{1}} \ldots \iota_{\epsilon_{N+1}} B_{X^{*}} \neq \emptyset$.

Next it is clear that we can pass to a further full subtree $T_{2}$ so that for every $a$ we have $\left|y^{*}\left(x_{a}\right)\right|<\delta$ (since $\left(x_{a}\right)$ is weakly null). Finally we use Lemma 3.3 to produce a full tree $T_{3} \subset T_{2}$ so that for any branch $\beta$

$$
\left|\left\langle\sum_{a \in \beta} x_{a}, \sum_{a \in \beta} x_{a}^{*}\right\rangle-\sum_{a \in \beta}\left\langle x_{a}, x_{a}^{*}\right\rangle\right| \leq \delta .
$$

Now for any branch $\beta$ in $T_{3}$ we have

$$
\left\|\sum_{a \in \beta} x_{a}\right\|=\left\langle\sum_{a \in \beta} x_{a}, y^{*}+\sum_{a \in \beta} x_{a}^{*}\right\rangle .
$$

Hence

$$
\begin{aligned}
1<\left\|\sum_{a \in \beta} x_{a}\right\| & \leq(N+2) \delta+\sum_{a \in \beta}\left\langle x_{a}, x_{a}^{*}\right\rangle \\
& \leq(2 N+3) \delta+2 \sigma \sum_{k=1}^{N+1} \epsilon_{k} .
\end{aligned}
$$

Thus we have $\sum_{k=1}^{N+1} \epsilon_{k} \geq \frac{1}{3} \sigma^{-1}$.

Theorem 4.4. Suppose $X$ is a separable Banach space containing no copy of $\ell_{1}$. Let $H(\tau)=(\mathrm{Cz}(X, \tau)-1)^{-1}$ for $0 \leq \tau<1$. Then there is a universal constant $C \leq 19200$ so that $N(\sigma)^{-1}$ is $C$-equivalent to $\varphi(\sigma)$ and $H(\tau)$ is $C$-equivalent to $\psi(\tau)$.

Proof. Suppose $0<\sigma<1$. Then by Lemma 2.4 and the preceding Lemma 4.3 we have that if $\tau \leq \frac{1}{12} \sigma^{-1}(N+1)^{-1}$, then

$$
\mathrm{Cz}(X, \tau)-1 \geq \frac{1}{24 \sigma \tau}
$$

Thus

$$
H(\tau) \leq 24 \sigma \tau
$$

Then, for any $\sigma$, if $0<\tau \leq \frac{25}{12} \sigma^{-1}(N(\sigma / 25)+1)^{-1}$ we have

$$
H^{*}(\sigma) \geq \sigma \tau-\frac{24}{25} \sigma \tau=\frac{1}{25} \sigma \tau
$$


Hence

$$
H^{*}(\sigma) \geq \frac{1}{12}(N(\sigma / 25)+1)^{-1} \geq \frac{1}{24}(N(\sigma / 25))^{-1} .
$$

Since $H^{*}$ is convex, this implies that $H^{*} 600$-dominates $N^{-1}$.

Now by Proposition 2.8 we have that $H$ 4-dominates $\psi$ and hence $\psi^{*} 4$-dominates $H^{*}$. Thus $\varphi C$-dominates $N^{-1}$ with $C \leq 19200$. By the remarks after Theorem 4.2 this means that $\varphi$ and $N^{-1}$ are $C$-equivalent.

Now recall that $H(n \tau) \geq n H(\tau)$ for $n \in \mathbb{N}$ by Lemma 2.2

Thus for $s \geq 1$ we have $H(s t) \geq \frac{1}{2} s H(t)$. It follows easily that $H$ is 2-equivalent to a function $H_{1}$ with the property that $H_{1}(t) / t$ is increasing, which is then 2equivalent to a convex function. Hence $H$ is 4 -equivalent to a convex function and hence also to $H^{* *}$.

Now $H^{*} 600$-dominates $N^{-1}$ and hence 1200 -dominates $\varphi$. Thus $H^{* *}$ is 1200 dominated by $\varphi^{*}$ and thus 4800 -dominated by $\psi$. Hence $H$ is 19200 -dominated by $\psi$, and so $H$ is 19200 -equivalent to $\psi$.

Theorem 4.5. Suppose $X$ is a separable Banach space not containing $\ell_{1}$. Then there is a universal constant $C<10^{6}$ so that if $0<\tau \leq 1$, then

$$
\mathrm{Cz}(X, \tau) \leq \sum_{\substack{k \geq 0 \\ 2^{k} \tau / C \leq 1}} 2^{k} \mathrm{Sz}\left(X, 2^{k} \tau / C\right) .
$$

Proof. Let

$$
K(\tau)=\left(\sum_{\substack{k \geq 0 \\ 2^{k} \tau \leq 1}} 2^{k}\left(\mathrm{Sz}\left(X, 2^{k} \tau\right)-1\right)\right)^{-1} .
$$

Then, arguing as with $H$, we have that $K$ is 4-equivalent to $K^{* *}$.

We next note that, by Lemma 4.3 and Lemma [2.4, if $0<\sigma<1$ we have that if $0<\tau \leq \frac{1}{6} \sigma^{-1}(N(\sigma)+1)^{-1}$ then

$$
K(\tau) \leq 6 \sigma \tau
$$

Reasoning as above gives

$$
K^{*}(\sigma) \geq \frac{1}{6}(N(\sigma / 7)+1)^{-1} \geq \frac{1}{12} N(\sigma / 7)^{-1} .
$$

Hence $K^{*}$ 100-dominates $N^{-1}$. By Theorem 4.4 this implies that $K$ is $C$ dominated by $H$ for a suitable absolute constant $C \leq 600.100 .4 .4<10^{6}$. This clearly yields the result.

Our next corollary follows easily from Theorem 4.5.

Corollary 4.6. Suppose $X$ is a separable Banach space with $\mathrm{Sz}(X)=\omega_{0}$. Suppose $p>1$ and

$$
\sup _{0<\epsilon<1} \epsilon^{p} \mathrm{Sz}(X, \epsilon)<\infty
$$

Then

$$
\sup _{0<\epsilon<1} \epsilon^{p} \mathrm{Cz}(X, \epsilon)<\infty .
$$


We are now in position to state our main renorming theorems. The first one simply restates Theorem 4.4 :

Theorem 4.7. Suppose $X$ is a separable Banach space with $\operatorname{Sz}(X)=\omega_{0}$. Then there exists an absolute constant $C \leq 19200$ such that for any $0<\tau<1$ there is a 2-equivalent norm $|\cdot|$ on $X$ so that if $x^{*}, x_{n}^{*} \in X^{*}$ satisfy $\left|x^{*}\right|=1,\left|x_{n}^{*}\right|=\tau$ and $\lim _{n \rightarrow \infty} x_{n}^{*}=0$ weak , then

$$
\liminf _{n \rightarrow \infty}\left|x^{*}+x_{n}^{*}\right| \geq 1+\frac{1}{\mathrm{Cz}(X, \tau / C)}
$$

It is clear that Theorem 4.7 is in a sense a best possible result. Notice that we do not have a simultaneous renorming which works for all $0<\tau<1$. However we can combine these norms to give a single norm which has the UKK ${ }^{*}$ property with a nearly optimal modulus. Note that it is clear that any space $X$ which has an equivalent $\mathrm{UKK}^{*}$ norm satisfies $\mathrm{Sz}(X)=\omega_{0}$.

Theorem 4.8. Suppose $X$ is a separable Banach space with $\mathrm{Sz}(X)=\omega_{0}$. Let $p_{X}$ be the Szlenk power type of $X$. Then for any $q>p_{X}$ there are an equivalent norm $|\cdot|$ on $X$ and $a$ constant $c>0$ so that if $0<\tau \leq 1$ and $x^{*}, x_{n}^{*} \in X^{*}$ satisfy $\left|x^{*}\right|=1$, $\left|x_{n}^{*}\right|=\tau$ and $\lim _{n \rightarrow \infty} x_{n}^{*}=0$ weak $k^{*}$ then

$$
\liminf _{n \rightarrow \infty}\left|x^{*}+x_{n}^{*}\right| \geq 1+c \tau^{q} .
$$

Proof. Fix $p_{X}<r<q$. In this case we have that $\mathrm{Sz}(X, \epsilon) \leq C \epsilon^{-r}$ for some constant $C$. By Corollary 4.6 we have a similar estimate $\mathrm{Cz}(X, \epsilon) \leq C \epsilon^{-r}$. Hence, for a suitable constant $c_{1}>0$, for each $k \in \mathbb{N}$ there is a norm $|\cdot|_{k}$ on $X$ which is 2-equivalent to the original norm and such that if $\left|x^{*}\right|_{k}=1$ and $\left|x_{n}^{*}\right|_{k}=2^{-k}$ with $x_{n}^{*}$ weak $^{*}$-null, then

$$
\liminf _{n \rightarrow \infty}\left|x^{*}+x_{n}^{*}\right|_{k} \geq 1+c_{1} 2^{-r k} .
$$

Now define the (dual) norm $|\cdot|$ on $X^{*}$ by

$$
\left|x^{*}\right|=\sum_{k=1}^{\infty} 2^{(r-q) k}\left|x^{*}\right|_{k} .
$$

This clearly defines an equivalent dual norm on $X^{*}$. Thus there is a uniform constant $B$ so that for every $k$ we have $B^{-1}\left|x^{*}\right| \leq\left|x^{*}\right|_{k} \leq B\left|x^{*}\right|$. Suppose $\left|x^{*}\right|=1$ and $\left|x_{n}^{*}\right|=\tau$ with $\left(x_{n}^{*}\right)$ weak $^{*}$-null. Pick $k \in \mathbb{N}$ so that $2^{-k} \leq B^{-2} \tau \leq 2^{1-k}$. Then $\left|x^{*}\right|_{k} \leq B$ and $\left|x_{n}^{*}\right|_{k} \geq B^{-1} \tau \geq 2^{-k}\left|x^{*}\right|_{k}$. Hence

$$
\liminf \left|x^{*}+x_{n}^{*}\right|_{k} \geq\left|x^{*}\right|_{k}\left(1+c_{1} 2^{-r k}\right) .
$$

This implies that

$$
\liminf \left|x^{*}+x_{n}^{*}\right| \geq 1+c_{2} 2^{-q k} \geq 1+c_{3} \tau^{q}
$$

for suitable $c_{2}, c_{3}>0$.

In general we do not know whether the functions $\mathrm{Cz}(X, \tau)$ and $\mathrm{Sz}(X, \tau)$ are equivalent. However in certain cases, which include for instance super-reflexive spaces and also James' quasi-reflexive space $J([21])$, they are equivalent:

Theorem 4.9. Suppose $X$ is a separable Banach space with $\mathrm{Sz}(X)=\operatorname{Sz}\left(X^{*}\right)=\omega_{0}$. Then there is a constant $C$ (depending only on $X$ ) so that $\mathrm{Cz}(X, \tau) \leq \mathrm{Sz}(X, \tau / C)$. 
Proof. We begin by noting that $X^{* *}$ is separable. From Theorem 4.7 and the fact that $\mathrm{Cz}\left(X^{*}, \tau\right)<\infty$ for all $\tau>0$ it follows that we can replace the original norm with an equivalent norm on $X \subset X^{* *}$ so that there exists $\delta>0$ with the property that if $\|x\|=\left\|x_{n}\right\|=1$ and $\left(x_{n}\right)$ is weakly null, then

$$
\liminf \left\|x+x_{n}\right\|>1+\delta
$$

Using this property, we make an estimate of $N(\sigma)$. If $N=N(\sigma)<\infty$, there is a weakly null tree-map $\left(x_{a}\right)_{a \in \mathcal{F} \mathbb{N}}$ of height $N+1$ so that $\left\|x_{a}\right\| \leq \sigma$ for every $a \in S$ but $\left\|\sum_{a \in \beta} x_{a}\right\|>1$ on every branch. We define a second tree-map $\left(y_{a}\right)_{a \in \mathcal{F} \mathbb{N}}$ by $y_{a}=x_{a}$ if $|a| \leq N+1$ and $y_{a}=x_{b}$ if $N+1<|a|=k \leq 2(N+1)$ and $b=\left\{m_{N+2}, \ldots, m_{k}\right\}$ if $a=\left\{m_{1}, \ldots, m_{k}\right\}$. If $|a|>2(N+1)$ then $y_{a}=0$. It follows easily from (4.1) that there is a full subtree $S$ so that on every branch we have $\sum_{a \in \beta}>1+\delta$. In particular, $N\left((1+\delta)^{-1} \sigma\right) \leq 2 N(\sigma)+1 \leq 3 N(\sigma)$.

Iterating we have $N(\sigma)<\infty$ for all $\sigma$. The estimate on $N$ clearly implies an estimate of the type

$$
N(\lambda \sigma) \leq C \lambda^{-p} N(\sigma), \quad 0<\lambda, \sigma<1,
$$

where $1<p<\infty$.

By Theorem 4.4 and Proposition 2.8 we obtain the dual estimate $\mathrm{Cz}(X, \lambda \tau) \geq$ $C \lambda^{-q} \mathrm{Cz}(X, \tau)$, where $\frac{1}{p}+\frac{1}{q}=1$. Note that $q>1$.

We now use Theorem 4.5 First note that for a suitable constant $C_{1}$ we have

$$
\begin{aligned}
\sum_{\substack{k \geq 0 \\
2^{k} \tau \leq 1}} 2^{k} \mathrm{Sz}\left(X, 2^{k} \tau\right) & \leq \sum_{\substack{k \geq 0 \\
2^{k} \tau \leq 1}} 2^{k} \mathrm{Cz}\left(X, 2^{k} \tau\right) \\
& \leq C \sum_{\substack{k \geq 0 \\
2^{k} \tau \leq 1}} 2^{k(1-q)} \mathrm{Cz}(X, \tau) \\
& \leq C_{1} \mathrm{Cz}(X, \tau) .
\end{aligned}
$$

Recall that we also have an estimate from Theorem 4.5

$$
\mathrm{Cz}(X, \tau) \leq \sum_{\substack{k \geq 0 \\ 2^{k} \tau / C \leq 1}} 2^{k} \mathrm{Sz}\left(X, 2^{k} \tau / C_{2}\right) .
$$

Now pick $k_{0}$ so that for every $\tau$ we have

$$
\mathrm{Cz}\left(X, 2^{-k_{0}} C_{2} \tau\right) \geq\left(1+2{ }^{k_{0}} C_{1}\right) \mathrm{Cz}(X, \tau) .
$$

This is possible by the growth condition on $\mathrm{Cz}(X, \tau)$ as $\tau \rightarrow 0$. Then

$$
\begin{aligned}
\mathrm{Cz}(X, \tau) & \leq \mathrm{Cz}\left(X, 2^{-k_{0}} C_{2} \tau\right)-2^{k_{0}} C_{1} \mathrm{Cz}(X, \tau) \\
& \leq \sum_{\substack{k \geq 0 \\
2^{k-k_{0}} \tau \leq 1}} 2^{k} \mathrm{Sz}\left(X, 2^{k-k_{0}} \tau\right)-\sum_{\substack{k \geq 0 \\
2^{k} \tau \leq 1}} 2^{k+k_{0}} \mathrm{Sz}\left(X, 2^{k} \tau\right) \\
& \leq \sum_{k=0}^{k_{0}-1} 2^{k} \mathrm{Sz}\left(X, 2^{k-k_{0}} \tau\right) \\
& \leq 2^{k_{0}} \mathrm{Sz}\left(X, 2^{-k_{0}} \tau\right) .
\end{aligned}
$$

This implies the theorem. 
Note that the above proof shows that if $X$ is separable and $\mathrm{Sz}(X)=\mathrm{Sz}\left(X^{*}\right)=$ $\omega_{0}$, then $N(\sigma)<\infty$ for any $\sigma>0$. Hence by Theorem 4.10 below, the Szlenk index of such spaces is not summable.

We conclude this section with a characterization of spaces with summable Szlenk index (see also Theorem 4.2). Recall that by Lemma 2.4. condition (iii) below is equivalent to the summability of the convex Szlenk index $\mathrm{Cz}$. Note that $\mathrm{Sz}$ and $\mathrm{Cz}$ are also equivalent for spaces which satisfy the conditions of the next theorem.

Theorem 4.10. Let $X$ be a separable Banach space. The following assertions are equivalent:

(i) $X$ has summable Szlenk index.

(ii) There exists $\sigma>0$ so that $N(\sigma)=\infty$.

(iii) There exists $K>0$ so that for any $0<\tau<1, \mathrm{Cz}(X, \tau) \leq K \tau^{-1}$.

(iv) For any function $f:(0,1) \rightarrow(0,1)$ which satisfies $\lim _{\tau \rightarrow 0} \tau^{-1} f(\tau)=0$, there are an equivalent norm $|\cdot|$ on $X$ and a constant $c>0$ so that if $0<\tau<1$ and $x^{*}, x_{n}^{*} \in X$ satisfy $\left|x^{*}\right|=1,\left|x_{n}^{*}\right|=\tau$ and $\lim _{n \rightarrow \infty} x_{n}^{*}=0$ weak $k^{*}$ then

$$
\liminf _{n \rightarrow \infty}\left|x^{*}+x_{n}^{*}\right| \geq 1+\operatorname{cf}(\tau) .
$$

Proof. We first note that (iii) $\Rightarrow$ (i) follows directly from the second part of Lemma 2.4 .

Next we prove that (iv) $\Rightarrow$ (iii). Assume that (iii) does not hold. Let

$$
f(\tau)=\sqrt{\frac{\tau}{\mathrm{Cz}(X, \tau)-1}} .
$$

Then we have $\lim _{\tau \rightarrow 0} \tau^{-1} f(\tau)=0$. Thus, assuming (iv), we deduce the estimate $\mathrm{Cz}(X, \tau) \leq C(f(\tau))^{-1}$, which gives a contradiction.

Next we note that (i) $\Rightarrow$ (ii) is immediate from Lemma 4.3

It remains therefore to show that (ii) $\Rightarrow$ (iv). Note first that we can assume that $\tau^{-1} f(\tau)$ is a monotone increasing function. It will be sufficient to prove the result with $f(\tau)$ replaced by $f(\tau / 4)$.

We note that by Theorem 4.4 and Proposition 2.8 we have that $\psi(\tau) \geq c \tau$ for some $c>0$. Hence if $0<c_{1}<c$ there is, for each $k \in \mathbb{N}$, a 2-renorming $|\cdot|_{k}$ of $X$ so that $\frac{1}{2}\|x\| \leq|x|_{k} \leq\|x\|$ for $x \in X$ and

$$
\liminf \left|x^{*}+x_{n}^{*}\right|_{k} \geq 1+c_{1} 2^{-k}
$$

whenever $\left|x^{*}\right|_{k}=1$ and $\left(x_{n}^{*}\right)$ is a weak*-null sequence in $X^{*}$ with $\left|x_{n}^{*}\right|_{k}=2^{-k}$. Now, using the same idea as in the preceding proof, we define a dual norm on $X^{*}$ by

$$
\left|x^{*}\right|=\frac{1}{2 f\left(\frac{1}{2}\right)} \sum_{k=1}^{\infty}\left(2^{k} f\left(2^{-k}\right)-2^{k+1} f\left(2^{-k-1}\right)\right)\left|x^{*}\right|_{k} .
$$

It follows from our definition that $|\cdot|$ is well-defined and 2-equivalent to the original norm. Now suppose $x^{*}, x_{n}^{*} \in X^{*}$ are such that $\left|x^{*}\right|=1,\left|x_{n}^{*}\right|=\tau$ and $\left(x_{n}^{*}\right)$ is weak ${ }^{*}$-null. Pick $j \in \mathbb{N}$ so that $2^{-j} \leq \frac{1}{4} \tau \leq 2^{1-j}$. Then if $k \geq j$ we have $\left|x^{*}\right|_{k} \leq 2$ and $\left|x_{n}^{*}\right|_{k} \geq \frac{1}{2} \tau \geq 2^{-j}\left|x^{*}\right|_{k}$.

Hence we obtain by convexity

$$
\liminf \left|x^{*}+x_{n}^{*}\right|_{k} \geq\left|x^{*}\right|_{k}\left(1+c_{1} 2^{-j}\right) .
$$


Summing over $k$, we have

$$
\liminf \left|x^{*}+x_{n}^{*}\right| \geq 1+c_{1} 2^{-j} \sum_{k=j}^{\infty}\left(2^{k} f\left(2^{-k}\right)-2^{k+1} f\left(2^{-k-1}\right)\right)\left|x^{*}\right|_{k} .
$$

Hence for a suitable constant $c_{2}>0$ we have

$$
\liminf \left|x^{*}+x_{n}^{*}\right| \geq 1+c_{2} f(\tau / 4) .
$$

This completes the proof.

Remarks. Spaces with summable Szlenk index are considered in [18], where it is shown that the original Tsirelson space is a reflexive example. These spaces are very close to being subspaces of $c_{0}$ which are characterized by the existence of an equivalent Lipschitz-UKK*-norm (cf. [17, 7]), i.e. a norm such that

$$
\liminf \left\|x^{*}+x_{n}^{*}\right\| \geq 1+c \tau
$$

whenever $\left\|x^{*}\right\|=1$ and $\left(x_{n}^{*}\right)$ is a weak ${ }^{*}$-null sequence with $\left\|x_{n}^{*}\right\|=\tau$. The "lack of isotropy" of the Szlenk derivation when applied to the Tsirelson space seems to be responsible for the difference between summability of the Szlenk index and embeddability into $c_{0}$.

\section{Applications to UNifORM AND LipsChitz hOMEOMORPhisms}

We first recall the Gorelik principle. We use the version proved in 7; see [14 for an earlier version. Both are based on the original idea of Gorelik [9]. If $V: X \rightarrow Y$ is uniformly continuous, we denote the modulus of continuity by

$$
\omega(V, t)=\sup \{\|V x-V y\| ;\|x-y\| \leq t\} .
$$

Proposition 5.1 (The Gorelik Principle). Let $X$ and $Y$ be two Banach spaces and let $U$ be a homeomorphism of $X$ onto $Y$ whose inverse is uniformly continuous. Suppose $b>0$ and $d>\omega\left(U^{-1}, b\right)$, and let $X_{0}$ be a closed subspace of $X$ of finite codimension. Then there is a compact subset $K$ of $Y$ so that

$$
b B_{Y} \subset K+U\left(2 d B_{X_{0}}\right) .
$$

Let us recall at this point that if $V: X \rightarrow Y$ is uniformly continuous, it is Lipschitz at large distances. More precisely, if $0<t<\infty$ then we have

$$
\|V x-V y\| \leq t^{-1} \omega(V, t)(t+\|x-y\|) .
$$

Let us define the asymptotic Lipschitz constant of $V$ by

$$
\|V\|_{u}=\lim _{t \rightarrow \infty} t^{-1} \omega(V, t) .
$$

If $X$ and $Y$ are uniformly homeomorphic Banach spaces, we define

$$
d_{u}(X, Y)=\inf \left\{\|U\|_{u}\left\|U^{-1}\right\|_{u}: U: X \rightarrow Y \text { is a uniform homeomorhism }\right\} .
$$

It is clear that $d_{u}(X, Y) \geq 1$, and that $d_{u}(X, Z) \leq d_{u}(X, Y) d_{u}(Y, Z)$ if $X, Y$ and $Z$ are all uniformly homeomorphic.

Lemma 5.2. Suppose $X, Y$ are uniformly homeomorphic. Then, if $M^{2}>d_{u}(X, Y)$ and $\eta>0$, there is a uniform homeomorphism $V: X \rightarrow Y$ so that we have

$$
\begin{aligned}
\left\|V x_{1}-V x_{2}\right\| & \leq M \max \left(\left\|x_{1}-x_{2}\right\|, \eta\right), & & x_{1}, x_{2} \in X, \\
\left\|V^{-1} y_{1}-V^{-1} y_{2}\right\| & \leq M \max \left(\left\|y_{1}-y_{2}\right\|, \eta\right), & & y_{1}, y_{2} \in Y .
\end{aligned}
$$


Proof. Let $U: X \rightarrow Y$ be a uniform homeomorphism with $\|U\|_{u}\left\|U^{-1}\right\|_{u}=L^{2}<$ $M^{2}$. For any $s>0$ define

$$
V_{s}(x)=L\|U\|_{u}^{-1} s^{-1} U(s x),
$$

so that

$$
V_{s}^{-1}(y)=s^{-1} U^{-1}\left(L^{-1}\|U\|_{u} s y\right) .
$$

Thus

$$
\omega\left(V_{s}, t\right)=L\|U\|_{u}^{-1} s^{-1} \omega(U, s t)
$$

and

$$
\omega\left(V_{s}^{-1}, t\right)=s^{-1} \omega\left(U^{-1}, L^{-1}\|U\|_{u} s t\right) .
$$

Hence for any $\delta>0$ we have

$$
\left\|V_{s} x_{1}-V_{s} x_{2}\right\| \leq L\|U\|_{u}^{-1} \delta^{-1} s^{-1} \omega(U, s \delta)\left(\delta+\left\|x_{1}-x_{2}\right\|\right)
$$

and

$$
\left\|V_{s}^{-1} y_{1}-V_{s}^{-1} y_{2}\right\| \leq \delta^{-1} s^{-1} \omega\left(U^{-1}, L^{-1}\|U\|_{u} s \delta\right)\left(\left\|y_{1}-y_{2}\right\|+\delta\right) .
$$

It is clear that if we take $s$ large enough and $\delta$ small enough, we obtain the lemma.

We now state our main result of this section:

Theorem 5.3. Suppose $X$ and $Y$ are separable Banach spaces which are uniformly homeomorphic. Then for any $\lambda>d_{u}(X, Y)$ and any $\epsilon>0$ there is a $\lambda$-equivalent norm $|\cdot|$ on $Y$ with

$$
\theta_{Y,|\cdot|}(\tau) \geq \theta_{X}(\tau / C)-\epsilon, \quad \forall 0 \leq \tau \leq 1,
$$

where $C=32 \lambda^{2}$.

Proof. Let $M=\lambda^{1 / 2}$. We assume that $U: X \rightarrow Y$ is a uniform homeomorphism of $X$ onto $Y$ which satisfies:

$$
\begin{array}{rlrl}
\left\|U x_{1}-U x_{2}\right\| & \leq M \max \left(\left\|x_{1}-x_{2}\right\|, 1\right), & & x_{1}, x_{2} \in X, \\
\left\|U^{-1} y_{1}-U^{-1} y_{2}\right\| & \leq M \max \left(\left\|y_{1}-y_{2}\right\|, 1\right), \quad & y_{1}, y_{2} \in Y .
\end{array}
$$

This is possible by Lemma 5.2. Recall that $C=32 \lambda^{2}=32 M^{4}$.

We first define a decreasing sequence of dual norms $\left\{|\cdot|_{k}\right\}_{k=1}^{\infty}$ on $Y^{*}$ by

$$
\left|y^{*}\right|_{k}=\sup \left\{\frac{\left|y^{*}\left(U x_{1}-U x_{2}\right)\right|}{\left\|x_{1}-x_{2}\right\|} ; \quad x_{1}, x_{2} \in X,\left\|x_{1}-x_{2}\right\| \geq 2^{k}\right\} .
$$

It is clear that we have $M^{-1}\left\|y^{*}\right\| \leq\left|y^{*}\right|_{k} \leq M\left\|y^{*}\right\|$ for every $k \geq 1$.

We will prove the following claim concerning the norms $|\cdot|_{k}$ :

Claim. Suppose $0<\tau<1$ and $y^{*}, y_{n}^{*} \in Y^{*}$ are such that $\left\|y^{*}\right\| \leq M,\left(y_{n}^{*}\right)$ is weak ${ }^{*}$-null and $\left\|y_{n}^{*}\right\| \geq M^{-1} \tau$ for all $n$. Then, if $2^{k} C \tau^{-1} \theta_{X}\left(C^{-1} \tau\right)>4 M$,

$$
\liminf _{n \rightarrow \infty}\left|y^{*}+y_{n}^{*}\right|_{k} \geq 2\left|y^{*}\right|_{k+1}-\left|y^{*}\right|_{k}+\theta_{X}(\tau / C) .
$$


To prove the claim we first define $\sigma=C \tau^{-1} \theta_{X}(\tau / C)$ so that $0<\sigma \leq 1$. Let us also for convenience of exposition write $\beta=\theta_{X}(\tau / C)$. Now for any $\epsilon>0$ we can choose $x, x^{\prime} \in X$ so that $\left\|x-x^{\prime}\right\| \geq 2^{k+1}$ and $y^{*}\left(U x-U x^{\prime}\right) \geq(1-\epsilon)\left\|x-x^{\prime}\right\|\left|y^{*}\right|_{k+1}$. As usual in such arguments, we can suppose by using translations that $x^{\prime}=-x$ and $U x^{\prime}=-U x$. Next we apply Proposition 2.6 and the separability of $X$. We deduce the existence of a finite codimensional subspace $X_{0}$ of $X$ so that

$$
\|x+z\| \geq\|x\| \geq 2^{k} \quad \forall z \in X_{0}
$$

and

$$
\|x+z\| \leq(1+2 \beta)\|x\| \quad \forall z \in \sigma\|x\| B_{X_{0}} .
$$

Now, by assumption we have $\omega\left(U^{-1}, b\right)<2 M b$ if $b \geq 1$. Hence we apply the Gorelik principle, Proposition 5.1 for $b=\frac{\sigma\|x\|}{4 M}>1$ by choice of $k$, and $d=\frac{\sigma\|x\|}{2}$. We deduce that there is a compact subset $K$ of $Y$ so that

$$
\frac{\sigma\|x\|}{4 M} B_{Y} \subset K+U\left(\sigma\|x\| B_{X_{0}}\right)
$$

It follows from (5.5) that there is a sequence $z_{n} \in \sigma\|x\| B_{X_{0}}$, so that

$$
\liminf _{n \rightarrow \infty} y_{n}^{*}\left(-U z_{n}\right) \geq \frac{\sigma \tau}{4 M^{2}}\|x\|=\frac{C \beta}{4 M^{2}}\|x\| .
$$

Now $\left\|x+z_{n}\right\|=\left\|z_{n}-x^{\prime}\right\|$, so that by (5.3) and (5.4) we have

$$
y^{*}\left(U x+U z_{n}\right)=y^{*}\left(U z_{n}-U x^{\prime}\right) \leq(1+2 \beta)\left|y^{*}\right|_{k}\|x\| .
$$

But

$$
y^{*}(U x)=\frac{1}{2} y^{*}\left(U x-U x^{\prime}\right) \geq(1-\epsilon)\left|y^{*}\right|_{k+1}\|x\| .
$$

Hence we deduce that

$$
y^{*}\left(U z_{n}\right) \leq\left[(1+2 \beta)\left|y^{*}\right|_{k}-(1-\epsilon)\left|y^{*}\right|_{k+1}\right]\|x\| .
$$

Combining these estimates gives

$$
\begin{aligned}
& \liminf _{n \rightarrow \infty}\left(y^{*}+y_{n}^{*}\right)\left(U x-U z_{n}\right) \\
& \quad \geq(2-2 \epsilon)\left|y^{*}\right|_{k+1}\|x\|-\left|y^{*}\right|_{k}\|x\|+\frac{C \beta}{4 M^{2}}\|x\|-2 \beta\left|y^{*}\right|_{k}\|x\| .
\end{aligned}
$$

The left-hand side is estimated by $(1+2 \beta) \liminf \left|y^{*}+y_{n}^{*}\right|_{k}\left\|x^{*}\right\|$. Hence, since $\epsilon>0$ is arbitrary and $\left\|y^{*}\right\| \leq M$,

$$
\begin{aligned}
\liminf _{n \rightarrow \infty}\left|y^{*}+y_{n}^{*}\right|_{k} & \geq(1-2 \beta)\left(2\left|y^{*}\right|_{k+1}-\left|y^{*}\right|_{k}+\frac{C-8 M^{3}}{4 M^{2}} \beta\right) \\
& \geq 2\left|y^{*}\right|_{k+1}-\left|y^{*}\right|_{k}+\frac{C-8 M^{3}-16 M^{4}}{4 M^{2}} \beta .
\end{aligned}
$$

Our choice of $C$ gives

$$
\frac{C-8 M^{3}-16 M^{4}}{4 M^{2}} \geq 1,
$$

and so the claim (5.2) is proved.

The completion of the argument from the claim is easy. If $\theta_{X}(1 / C) \leq \epsilon$, the original norm will suffice. Otherwise choose $\tau_{0}$ so that $\theta_{X}\left(\tau_{0} / C\right)=\epsilon$. 
Pick $k_{0} \in \mathbb{N}$ so that $\frac{C}{\tau_{0}} \theta_{X}\left(\frac{\tau_{0}}{C}\right) 2^{k_{0}}>4 M$. Pick an integer $N$ so that

$$
\frac{2 M^{2}}{N}<\epsilon
$$

Now let

$$
\left|y^{*}\right|=\frac{1}{N} \sum_{k=k_{0}+1}^{k=k_{0}+N}\left|y^{*}\right|_{k}
$$

which clearly defines a dual norm on $Y^{*}$ with $M^{-1}\left\|y^{*}\right\| \leq\left|y^{*}\right| \leq M\left\|y^{*}\right\|$.

Assume $\left|y^{*}\right|=1$ and that $\left(y_{n}^{*}\right)$ is weak* null with $\left|y_{n}^{*}\right| \geq \tau$. Then $\left\|y^{*}\right\| \leq M$ and $\left\|y_{n}^{*}\right\| \geq \tau / M$. Hence for each $k_{0}+1 \leq k \leq k_{0}+N$ we have (5.2). Summing gives

$$
\liminf \left|y^{*}+y_{n}^{*}\right| \geq\left|y^{*}\right|-\frac{2}{N}\left|y^{*}\right|_{k_{0}+1}+\theta_{X}(\tau / C) \text {. }
$$

Now

$$
\frac{2}{N}\left|y^{*}\right|_{k_{0}+1} \leq \frac{2 M}{N}\left\|y^{*}\right\| \leq \frac{2 M^{2}}{N}<\epsilon,
$$

so that the theorem is proved.

Before turning to applications for this result, let us notice that in the case of Lipschitz equivalence the same techniques can be applied to give a rather stronger result. A special case of the theorem below is shown in [7.

Theorem 5.4. Suppose $X$ and $Y$ are separable Banach spaces which are Lipschitz isomorphic. Then there is an equivalent norm on $Y$ so that $\theta_{Y} C$-dominates $\theta_{X}$ for some $C>0$.

Proof. We only provide a sketch. In this case one can use the norm on $Y^{*}$ defined by

$$
\left|y^{*}\right|=\sup \left\{\frac{\left|y^{*}\left(U x_{1}\right)-y^{*}\left(U x_{2}\right)\right|}{\left\|x_{1}-x_{2}\right\|}, x_{1} \neq x_{2}\right\} .
$$

The calculations are similar but rather simpler. We leave the details to the reader.

Our main application of Theorem 5.3 is the fact that the convex Szlenk index is (up to equivalence) invariant under uniform homeomorphisms.

Theorem 5.5. Suppose $X$ and $Y$ are uniformly homeomorphic. Then:

(i) $\mathrm{Sz}(X) \leq \omega_{0}$ if and only if $\mathrm{Sz}(Y) \leq \omega_{0}$.

(ii) There exists a constant $C$ so that if $0 \leq \tau \leq 1$, then

$$
\mathrm{Cz}(X, C \tau) \leq \mathrm{Cz}(Y, \tau) \leq \mathrm{Cz}(X, \tau / C) .
$$

Proof. We note first that the relevant functionals are separably determined ([19]). Hence we may assume that $X$ and $Y$ are separable. The result is then an almost immediate deduction from Theorem 5.3 In fact it is immediately clear that the statement of the theorem yields, that for the original norm on $Y$,

$$
\mathrm{Cz}(Y, \tau) \leq\left(\theta_{X}\left(\frac{\tau}{C \lambda}\right)\right)^{-1}+1 .
$$

If we then consider all 2-equivalent norms on $X$, we deduce an estimate of the form

$$
\mathrm{Cz}(Y, \tau) \leq \psi_{X}(\tau / C)^{-1}+1
$$


for a different constant $C$. However, by Theorem 4.7 this implies an estimate

$$
\mathrm{Cz}(Y, \tau) \leq \mathrm{Cz}(X, \tau / C) .
$$

This estimate and its converse establish both (i) and (ii).

Theorem 5.6. (i) Let $X$ be a Banach space which is uniformly homeomorphic to a subspace of $c_{0}$. Then $X$ has summable Szlenk index (and in particular, $X^{*}$ is separable).

(ii) If $X$ is uniformly isomorphic to $c_{0}$, then $X^{*}$ is linearly isomorphic to $\ell_{1}$.

Proof. Since any subspace of $c_{0}$ has summable Szlenk index, then $X$ must also have summable Szlenk index by Theorem 5.5 and Theorem 4.10 For the second part note that $X$ must be an $\mathcal{L}_{\infty}$-space [11, and so (i) yields that $X^{*}$ is isomorphic to $\ell_{1}[22$.

Remarks. 1) It is shown in [1] that the Bourgain-Delbaen preduals of $l^{1}$ have a finite Szlenk index, although they do not contain $c_{0}$. On the other hand, it is shown in [10] that these spaces contain $l^{p}$ hereditarily for some $p \in(1, \infty)$. It follows now from Theorem 5.6 that no infinite-dimensional subspace of a Bourgain-Delbaen space is uniformly homeomorphic to a subspace of $c_{0}$.

2)As remarked above, there are unfortunately examples of Banach spaces with summable Szlenk index which do not embed into $c_{0}$, as shown in [18]. We conjecture, however, that any predual of $\ell_{1}$ with summable Szlenk index is isomorphic to $c_{0}$.

A Banach space which is Lipschitz isomorphic to $c_{0}$ is (in the separable case) linearly isomorphic to $c_{0}$ ([7). We do not know whether a space which is uniformly homeomorphic to $c_{0}$ is isomorphic to $c_{0}$. Curiously, however, we can show that if $d_{u}\left(X, c_{0}\right)$ is small enough, then $X$ is isomorphic to $c_{0}$. This result is very similar to the quantitative Lipschitz result in [7. Note that the following statement is true for any equivalent renorming of $c_{0}$, although the function $f$ and $\epsilon_{0}$ depend upon the given norm.

Theorem 5.7. There exist $\epsilon_{0}>0$ and a function $f:\left(0, \epsilon_{0}\right] \rightarrow(0, \infty)$ with $\lim _{\epsilon \rightarrow 0} f(\epsilon)=0$ so that if $d_{u}\left(X, c_{0}\right)<1+\epsilon$, then $d\left(X, c_{0}\right)<1+f(\epsilon)$.

Proof. We use the ideas of [16]; in particular, we use the the Gromov-Hausdorff distance for two Banach spaces $d_{G H}(X, Y)$, for which we refer to [16. From Theorem 5.9 of [16] we have the fact that if $X_{n}$ is a sequence of Banach spaces converging to $c_{0}$ in $d_{G H}$, then the Banach-Mazur distance $d\left(X_{n}, c_{0}\right) \rightarrow 1$. It therefore suffices to show that $d_{u}\left(X_{n}, c_{0}\right) \rightarrow 1$ implies $d_{G H}\left(X, c_{0}\right) \rightarrow 0$.

To do this, suppose that $d_{u}(X, Y)<1+\epsilon$. Then for any $\eta>0$ we use Lemma 5.2 to produce a uniform homeomorphism $V: X \rightarrow Y$ such that $V(0)=0$ and

$$
\begin{aligned}
\left\|V x_{1}-V x_{2}\right\| & \left.\leq(1+\epsilon)^{1 / 2}\right) \max \left(\left\|x_{1}-x_{2}\right\|, \eta\right), \\
\left\|V^{-1} y_{1}-V^{-1} y_{2}\right\| & \left.\leq(1+\epsilon)^{1 / 2}\right) \max \left(\left\|y_{1}-y_{2}\right\|, \eta\right), \quad x_{2}, \quad y_{1}, y_{2} \in Y .
\end{aligned}
$$

Now define $\Phi: B_{X} \rightarrow B_{Y}$ by $\Phi(x)=(1+\epsilon)^{-1 / 2} V x$ and $\Psi: B_{Y} \rightarrow B_{X}$ by $\Psi(y)=(1+\epsilon)^{-1 / 2} V^{-1} y$.

Then, if $x \in B_{X}, y \in B_{Y}$,

$$
\left|\|x-\Psi(y)\|-\left\|x-V^{-1}(y)\right\|\right| \leq \frac{1}{2} \epsilon
$$


and

$$
|\|\Phi(x)-y\|-\|V x-y\|| \leq \frac{1}{2} \epsilon .
$$

Now

$$
\|V x-y\| \leq(1+\epsilon)^{1 / 2}\left(\left\|x-V^{-1} y\right\|+\eta\right),
$$

and a similar reverse inequality gives

$$
\left|\|V x-y\|-\left\|x-V^{-1} y\right\|\right| \leq \frac{1}{2} \epsilon\left(\max \left\|x-V^{-1} y\right\|,\|V x-y\|\right)+2 \eta .
$$

Since $\left\|x-V^{-1} y\right\|,\|V x-y\| \leq(1+\epsilon)^{1 / 2}+1<3$, we obtain

$$
|\|x-\Psi(y)\|-\|\Phi(x)-y\||<\frac{5}{2} \epsilon+2 \eta .
$$

This implies ([16]) that $d_{G H}(X, Y) \leq \frac{5}{4} \epsilon+\eta$. The result then follows.

We now give one more application of our main result on the invariance of the convex Szlenk index:

Theorem 5.8. Suppose $2<p<\infty$ and $X$ is a quotient (resp. subspace) of $\ell_{p}$. If $Y$ is uniformly homeomorphic to $X$, then $Y$ is linearly isomorphic to a quotient (resp. subspace) of $\ell_{p}$.

Remark. In fact the methods of 14] give the subspace version rather easily, so the quotient case is the more interesting.

Proof. In both cases we have $\mathrm{Cz}(X, \epsilon) \leq C \epsilon^{-q}$, where $\frac{1}{p}+\frac{1}{q}=1$. We therefore deduce by Theorem 5.5 that $\mathrm{Cz}(Y, \epsilon) \leq C \epsilon^{-q}$.

Now we use the "standard ultraproduct technique" (cf. [14, p. 438, or [3]). The spaces $X$ and $Y$ are super-reflexive, and hence we can find Lipschitz-isomorphic separable spaces $X_{1} \supset X, Y_{1} \supset Y$ so that $X_{1}$ is one-complemented in an ultraproduct $X_{\mathcal{U}}$ and $Y_{1}$ is one-complemented in $Y_{\mathcal{U}}$. Then $Y_{1}$ embeds complementably into $X_{1}$ and $X_{1}$ embeds complementably in $Y_{1}$. Since $X$ and $Y$ are complemented in their ultraproducts, this leads to the fact that $Y$ is a quotient (respectively a subspace) of $L_{p}$.

In the case when $X$ is a subspace of $\ell_{p}$ we can complete the argument very simply. Since $p>2$, if $Y$ does not embed into $\ell_{p}$ then $\ell_{2}$ embeds complementably in $Y$ [15], and so $\mathrm{Cz}(X, \epsilon) \geq c \epsilon^{-2}$ for some $c>0$, which yields a contradiction.

Now consider the case when $X$ is a quotient of $\ell_{p}$, so that $Y$ is a quotient of $L_{p}[0,1]$. We have $\mathrm{Cz}(Y, \epsilon) \leq C \epsilon^{-q}$. It follows from Theorem 4.4 and Proposition 2.8 that $\psi_{Y}(\tau) \leq C_{1} \epsilon^{p}$ for a suitable constant $C_{1}$. We will argue that this implies $Y$ is of type $p$-Banach-Saks in the sense of [12, i.e. there is a constant $\lambda>0$ so that every normalized weakly null basic sequence $\left(w_{n}\right)_{n=1}^{\infty}$ has a subsequence $\left(v_{n}\right)$ satisfying $\left\|\sum_{i=1}^{n} v_{i}\right\| \leq \lambda n^{1 / p}$.

To do this we note that by the definition of $\psi_{Y}$ there is, for each $k \in \mathbb{N}$, an equivalent norm $|\cdot|_{k}$ on $Y$ so that $\|y\| \leq|y|_{k} \leq 2\|y\|$ for $y \in Y$ and

$$
\limsup \left|y+y_{n}\right|_{k}^{p} \leq|y|_{k}^{p}\left(1+2 C_{1} k^{-1}\right)
$$

if $\left(y_{n}\right)$ is weakly null and $\sup \left|y_{n}\right|_{k} \leq k^{-1 / p}|y|_{k}$. 
Now if $\left(w_{n}\right)$ is a normalized weakly null basic sequence in $(Y,\|\cdot\|)$, we pass to a subsequence $\left(v_{n}\right)$ such that if $1 \leq k \leq 2^{n}$ is such that $\left|v_{n}\right|_{k} \leq k^{-1 / p}\left|\sum_{i=1}^{n-1} v_{i}\right|_{k}$ then

$$
\left|\sum_{i=1}^{n} v_{i}\right|_{k} \leq\left|\sum_{i=1}^{n-1} v_{i}\right|_{k}\left(1+3 C_{1} k^{-1}\right)^{1 / p} .
$$

For fixed $n$, let $m=\left[\log _{2} n\right]$. Let $r$ be the greatest integer with $1 \leq r \leq n$ so that $\left|v_{r}\right|_{n}>n^{-1 / p}\left|\sum_{i=1}^{r-1} v_{i}\right|_{n}$. Provided $1 \geq 2 n^{-1 / p}\left(\log _{2} n+1\right)$, we have $r>m$. Hence for $r \leq j \leq n-1$ we have

$$
\left|\sum_{i=1}^{j+1} v_{i}\right|_{n} \leq\left(1+3 C_{1} n^{-1}\right)^{1 / p}\left|\sum_{i=1}^{j} v_{i}\right|_{n}
$$

and so

$$
\begin{aligned}
\left\|\sum_{i=1}^{n} v_{i}\right\| & \leq\left|\sum_{i=1}^{n} v_{i}\right|_{n} \leq\left(1+3 C_{1} n^{-1}\right)^{n / p}\left|\sum_{i=1}^{r} v_{i}\right|_{n} \\
& \leq e^{3 C_{1} / p}\left(\left|\sum_{i=1}^{r-1} v_{i}\right|_{n}+\left|v_{r}\right|_{n}\right) \\
& \leq e^{3 C_{1} / p}\left(n^{1 / p}+1\right)\left|v_{r}\right|_{n} \\
& \leq 4 e^{3 C_{1} / p} n^{1 / p}
\end{aligned}
$$

We can now combine Theorems III.1 and III.2 (or Remark III.3) of [12] to deduce that $Y$ is isomorphic to a quotient of $\ell_{p}$.

We refer to [5] for a similar result for spaces which are Lipschitz-isomorphic to linear quotients of $c_{0}$.

\section{REFERENCES}

[1] D. Alspach, The dual of the Bourgain-Delbaen space, Israel J. Math. 117 (2000) 239-259. MR 2001d:46022

[2] D. Alspach, R. Judd and E. Odell, The Szlenk index and local $l_{1}$ indices of a Banach space, to appear.

[3] Y. Benyamini, The uniform classification of Banach spaces, Longhorn Notes, University of Texas Austin, 1984-5, pp. 15-38. MR 87a:46007

[4] S.J. Dilworth, M. Girardi and D. Kutzarova, Banach spaces which admit a norm with the uniform Kadec-Klee property, Studia Math. 112 (1995) 267-277. MR 96a:46023

[5] Y. Dutrieux, Quotients of $c_{0}$ and Lipschitz-homeomorphisms, Houston J. Math., to appear.

[6] P. Enflo, Banach spaces which can be given an equivalent uniformly convex norm, Israel J. Math. 13 (1972) 281-288. MR 49:1073

[7] G. Godefroy, N.J. Kalton and G. Lancien, Subspaces of $c_{0}(\mathbb{N})$ and Lipschitz isomorphisms, Geom. Funct. Anal. 10 (2000) 798-820. CMP 2001:03

[8] G. Godefroy, N.J. Kalton and G. Lancien, L'espace de Banach $c_{0}$ est déterminé par sa métrique, C. R. Acad. Sci. Paris Sér. I Math. 327 (1998) 817-822. MR 99h:46007

[9] E. Gorelik, The uniform non-equivalence of $L_{p}$ and $\ell_{p}$, Israel J. Math. 87 (1994) 1-8. MR 95f:46028

[10] R. Haydon, Subspaces of the Bourgain-Delbaen space, Studia Math. 139 (2000) 275-293. CMP 2000:13

[11] S. Heinrich and P. Mankiewicz, Applications of ultrapowers to the uniform and Lipschitz classification of Banach spaces, Studia Math. 73 (1982) 225-251. MR 84h:46026

[12] W.B. Johnson, On quotients of $L^{p}$ which are quotients of $l^{p}$, Compositio Mathematica, 34 (1977) 69-89. MR 56:12844 
[13] W.B. Johnson, J. Lindenstrauss, D. Preiss and G. Schechtman, Almost Fréchet differentiability of Lipschitz mappings between infinite dimensional Banach spaces, Preprint (2000).

[14] W.B. Johnson, J. Lindenstrauss and G. Schechtman, Banach spaces determined by their uniform structure, Geom. Funct. Anal. 3 (1996) 430-470. MR 97b:46016

[15] W.B. Johnson and E. Odell, Subspaces of $L_{p}$ which embed into $\ell_{p}$, Compositio Math. 28 (1974) 37-49. MR 50:5424

[16] N.J. Kalton and M.M. Ostrovskii, Distances between Banach spaces, Forum Math. 11(1999), 17-48. MR 2000c:46024

[17] N.J. Kalton and D. Werner, Property (M), M-ideals and almost isometric structure, J. Reine Angew. Math. 461 (1995) 137-178. MR 96m:46022

[18] H. Knaust, E. Odell and T. Schlumprecht, On asymptotic structure, the Szlenk index and UKK properties in Banach spaces, Positivity 3 (1999) 173-199. CMP 99:16

[19] G. Lancien, Théorie de l'indice et problèmes de renormage en géometrie des espaces de Banach, Thèse de doctorat de l'Université Paris VI, January 1992.

[20] G. Lancien, On uniformly convex and uniformly Kadec-Klee renormings, Serdica Math. J. 21 (1995) 1-18. MR 96e:46009

[21] G. Lancien, Réflexivité et normes duales possédant la propriété uniforme de Kadec-Klee, Publ. Math. de la Fac. des sciences de Besançon, Fasicule 14 (1993-94) 69-74.

[22] D.R. Lewis and C. Stegall, Banach spaces whose duals are isomorphic to $\ell_{1}(\Gamma)$, J. Functional Analysis 12 (1973) 177-187. MR 49:7731

[23] J. Lindenstrauss and L. Tzafriri, Classical Banach spaces, Vol. 1, Sequence spaces, SpringerVerlag, 1977. MR 58:17766

[24] V. Milman, Geometric theory of Banach spaces. II. Geometry of the unit ball (Russian) Uspehi Mat. Nauk 26 (1971), 6 (162), 73-149. English translation: Russian Math. Surveys 26 (1971), 6, 79-163. MR 54:8240

[25] E. Odell and H.P. Rosenthal, A double dual characterization of separable Banach spaces containing $\ell$, Israel J. Math. 20 (1975) 375-384. MR 51:13654

[26] G. Pisier, Martingales with values in uniformly convex spaces, Israel J. Math. 20 (1975), 326-350. MR 52:14940]

[27] M. Ribe, Existence of separable uniformly homeomorphic non-isomorphic Banach spaces, Israel J. Math. 48 (1984), 139-147. MR 86e:46015

[28] W. Szlenk, The non-existence of a separable reflexive Banach space universal for all separable reflexive Banach spaces, Studia Math. 30 (1968) 53-61. MR 37:3327

Equipe d’Analyse, Université Paris Vi, Boite 186, 4, Place Jussieu, 75252 Paris Cedex 05, France

E-mail address: gig@ccr.jussieu.fr

Department of Mathematics, University of Missouri-Columbia, Columbia, Missouri 65211

E-mail address: nigel@math.missouri.edu

Equipe de Mathématiques - UMR 6623, Université de Franche-Comté, F-25030 BesanÇON CEDEX

E-mail address: GLancien@vega.univ-fcomte.fr 